\title{
Rounding Effects of Quenched Randomness on First-Order Phase Transitions
}

\author{
Michael Aizenman ${ }^{1 \mathrm{a}, \mathrm{b}}$ and Jan Wehr ${ }^{2 \mathrm{~b}, \mathrm{c}}$ \\ 1 Courant Institute of Mathematical Sciences, New York University, 251 Mercer St., New York, NY \\ 10012, USA \\ 2 School of Mathematics, Institute for Advanced Study, Princeton, New Jersey 08540, USA
}

Dedicated to R. L. Dobrushin, on the occasion of his $60^{\text {th }}$ birthday

\begin{abstract}
Frozen-in disorder in an otherwise homogeneous system, is modeled by interaction terms with random coefficients, given by independent random variables with a translation-invariant distribution. For such systems, it is proven that in $d=2$ dimensions there can be no first-order phase transition associated with discontinuities in the thermal average of a quantity coupled to the randomized parameter. Discontinuities which would amount to a continuous symmetry breaking, in systems which are (stochastically) invariant under the action of a continuous subgroup of $O(N)$, are suppressed by the randomness in dimensions $d \leqq 4$. Specific implications are found in the Random-Field Ising Model, for which we conclude that in $d=2$ dimensions at all $(\beta, h)$ the Gibbs state is unique for almost all field configurations, and in the Random-Bond Potts Model where the general phenomenon is manifested in the vanishing of the latent heat at the transition point. The results are explained by the argument of Imry and Ma [1]. The proofs involve the analysis of fluctuations of free energy differences, which are shown (using martingale techniques) to be Gaussian on the suitable scale.
\end{abstract}

\section{Table of Contents}

1. Introduction . . . . . . . . . . . . . . . . . . . . 490

a. The Rounding Effect . . . . . . . . . . . . . . . . 490

b. Outline of the Argument . . . . . . . . . . . . . . . . . . 492

2. Examples: Random-Field, Random-Bond, and Spin Glass Models. . 493

3. General Systems with Quenched Randomness. . . . . . . . . 495

a. The Setup . . . . . . . . . . . . . . . . . . . . 495

b. Systems with Long Range Interactions . . . . . . . . . . 498

\footnotetext{
a Also in the Physics Department

b Research partially supported by NSF grants PHY-8896163 and PHY-8912067

c Work done in part at Rutgers University, Department of Mathematics
} 
4. Statement of the Main Results . . . . . . . . . . . . . . 499

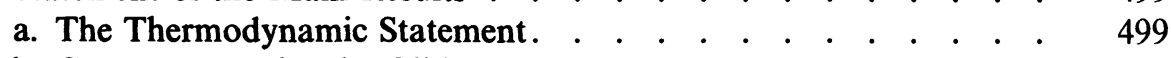

b. Consequences for the Gibbs States. . . . . . . . . . . . . . 500

c. Systems with Continuous Symmetry . . . . . . . . . . . 501

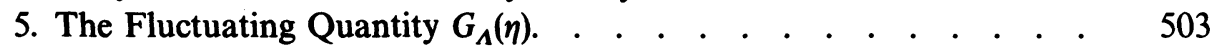

6. Proof of the Rounding of First-Order Phase Transitions in $d \leqq 2$ Dimensions. . . . . . . . . . . . . . . . . . . 507

7. The Rounding Effect in Systems with Continuous Symmetries in $d \leqq 4$ Dimensions. . . . . . . . . . . . . . . . . . . 511

Appendix I: Gibbs States for Random Systems . . . . . . . . . 516

a. Topological Preliminaries . . . . . . . . . . . . . . . 516

b. The Construction of $\mu_{\eta}$. . . . . . . . . . . . . . . . . . . . . . . . . . 518

Appendix II: A General Lower Bound for the Moment General Function 521

Appendix III: Variance Lower Bounds for Probability Measures on $\mathbb{R}^{N} \quad 523$

a. Measures on $\mathbb{R}$. . . . . . . . . . . . . . . . . . . . . . . . 523

b. Measures on $\mathbb{R}^{\mathbf{N}}$. . . . . . . . . . . . . . . . . . . . . . 525

References . . . . . . . . . . . . . . . 526

\section{Introduction}

a. The Rounding Effect. Frozen-in disorder is now recognized to have the potential to drastically alter some physically interesting properties of homogeneous media [2]. Even when the effect on a bulk property is perturbatively small, for low densities-or intensities of the disorder, the perturbation theory is typically nontrivial, as the disorder may result in deep structural changes. It may also happen that a sharp qualitative deviation from the behavior found in a homogeneous system occurs at any positive density of the disorder. The nature of the response of the system to the presence of disorder may depend on the dimension, with the latter behavior typically occurring in the lower dimensions. The systems studied here are of that kind.

Mathematical models with disorder which have been of interest in physics include: a) statistical mechanical models with random parameters, such as the models discussed in this work, and also b) Schrödinger operators with periodic plus random potentials [studied in relation to the electrical conductivity in the solid state], and c) random walks (simple, and self avoiding) in random environments. Some of the vast literature on these topics may be seen by consulting the references cited in [2]. Though our results are confined to the first case, the methods presented here-which consist of the analysis of fluctuations, may have some relevance also for the other situations.

In models of statistical mechanics, when the thermal averages are taken at fixed values of the random parameters - as is done when describing the thermal properties of systems with impurities of relatively long relaxation times, the randomness is referred to as quenched. This work is focused on the effect of quenched disorder on first-order phase transitions. Such a transition has a dual manifestation - in a discontinuity of one of the first order partial derivatives of the free energy, 
and in the nonuniqueness of the infinite-volume Gibbs state. Our main result, which was reported in [3], is a rigorous proof that in $d \leqq 2$ dimensions the presence of random (independent) fluctuations in the structural parameters results in the suppression of first-order phase transitions associated with discontinuities in the densities of the quantities conjugate to the randomized parameters. Discontinuities related to continuous symmetry breaking, as in the Heisenberg model at vanishing external field, are eliminated by arbitrarily weak quenched disorder (with a rotation-invariant distribution) even in the higher dimensions: $d \leqq 4$. The dimensions covered by the above results include the marginal cases.

Particular manifestation of the rounding of first-order transitions in 2D is seen in the ferromagnetic random-field Ising spin models (RFIM), around which many of the ideas related to the subject have been developed. For the RFIM the converse statement, i.e. that in the dimensions $d \geqq 3$ a first-order phase transition persists at weak enough disorder, has now been rigorously established $[5,6]$. The early prediction, and an insightful explanation of the effects of quenched randomness, was given by Imry and $\mathrm{Ma}$ [1], on the basis of the heuristic argument that the state of a system which is about to undergo a first-order transition is determined by the competition of the random-field, whose cumulative effect on a uniform spin configuration in a region $\Lambda=[-L, L]^{d}$ is of the order $\sqrt{|\Lambda|}=L^{d / 2}$, with the symmetry breaking mechanism whose strength is of the order of the boundary $|\partial \Lambda|=L^{d-1}$. For the marginal case of $d=2$ dimensions, the prediction has been that the fluctuating field will have the dominant effect. In the presence of a continuous symmetry, soft modes reduce the effect of the boundary conditions to $L^{d-2}$, and hence the marginal dimension is $d=4$.

Though the Imry-Ma argument is apparently based on the most relevant considerations, it was found to be insufficient when a need arose to decide between its prediction and that of a "dimensional reduction" principle, which suggested a higher value for the lower critical dimension of the RFIM. The resolution of the impasse required a series of rigorous works - starting with [4], and culminating with Imbrie [5] and Bricmont and Kupiainen [6], which prove the stability (under weak disorder) or the Peierls's mechanism (of symmetry breaking in the ground state [5], and at low temperatures [6]) in $d \geqq 3$ dimensions. The methods employed there could not, however, establish the disappearance of the symmetry breaking in $2 \mathrm{D}$, which the results reported here prove for arbitrarily weak random fields. Though that part of the Imry-Ma prediction was not challenged by the alternative argument, it has been argued that it also deserves rigorous study-in particular since it concerns a qualitatively distinct nonperturbative effect.

Another example of the rounding of a first-order phase transition is the suppression of the discontinuity in the energy density [7] (i.e. elimination of the latent heat) of the random-bond Potts models. These systems continue to exhibit some form of a phase transition (between an ordered and a disordered phase) even in the aftermath of the rounding effect [8]. Whether any phase transition persists in the RFIM is still an unresolved question [9].

The vanishing of the latent heat in the random-bond Potts model was also noted, independently of [3], in a work by K. Hui and A. N. Berker [10], where it is derived-along with some further information on the nature of the phase 
transition - by renormalization group (non-rigorous) arguments. There is currently a growing interest in related effects at both theoretical and experimental levels $[10,11]$.

b. Outline of the Argument. Our analysis is based on the consideration of fluctuations of some relevant functions of the random parameters, denoted here by $\left\{\varepsilon \eta_{x}\right\}$ ( $\varepsilon$ being a uniform factor setting the effective scale of the randomness and $\eta_{x}$ having variance 1 ), in a scheme which may be useful also for other problems.

The jump of the free energy's first derivative, $M=\frac{1}{2}((\partial F / \partial h+)-(\partial F / \partial h-))$, is realized as the average over the random parameters of the difference in the thermal expectation values of a conjugate quantity (single-site spin for random field models, and more generally the Hamiltonian's derivative with respect to $\eta_{x}$ ), between a pair of Gibbs measures generalizing the Ising model's \pm states. We introduce an auxiliary quantity, $G_{A}$ (whose definition for the general case requires some technical discussion), which expresses the difference in the finite-volume free energies of such a pair of states. The argument proceeds by showing that $M \neq 0$ carries the implication that for a set of random couplings of nonvanishing probability, the value of the related quantity $\tilde{G}_{A}\left(\eta_{A}\right)=E\left(G_{A} \mid\left\{\eta_{x}\right\}_{x \in A}\right)-E\left(G_{A}\right)$ (where $E(\cdot)$ is the conditional expectation) exceeds an upper bound set by simple energy estimates.

The fundamental relation of $G_{A}$, and $\tilde{G}_{A}$, with $M$ is:

$$
A v\left(\frac{\partial}{\partial \eta_{x}} \tilde{G}_{\Lambda}\right)=\varepsilon M, \text { for each } x \text { in } \Lambda \text {. }
$$

This implies that if $M \neq 0$ and $\varepsilon \neq 0$, then $\tilde{G}_{A}$ is "truly" dependent on $\approx|\Lambda|$ independently fluctuating quantities, and hence it should reach the order of magnitude of $\sqrt{|\Lambda|}$. That observation is contrasted with the simple free energy bound: $\widetilde{G}_{\Lambda} \leqq$ Const. $|\partial \Lambda|$ (for systems with short-range interactions). In $d=2$ dimensions these statements are consistent only if either $M=0$, or if the distribution of the quantity $\tilde{G}_{A}$ manifests a sharp cutoff on the scale of its typical order of magnitude. That feat should be rather difficult, given the extensive nature of $\tilde{G}_{\Lambda}$. To reach a contradiction, which then implies that in $d \leqq 2$ dimensions $M=0$, it is required to strengthen the basic observation on the size of $\widetilde{G}_{A}$ by additional information on the probability of large fluctuations of $\tilde{G}_{\Lambda} / \sqrt{|\Lambda|}$.

The argument outlined above is actually carried out by establishing the following pair of conflicting properties for the quantity $\tilde{G}_{A}$ (with $\Lambda$ ranging over $d$-dimensional cubes of linear size $L \rightarrow \infty$ ):

i. a Central Limit behavior:

$$
\tilde{G}_{\Lambda} / \sqrt{|\Lambda|} \rightarrow N(0, b) \text { (in distribution), with } b>0 \text { when } M \neq 0,
$$

ii. a uniform bound

$$
\left|\tilde{G}_{\Lambda}\left(\eta_{\Lambda}\right)\right| \leqq A|\partial \Lambda|+B \sqrt{|\Lambda|} \text { with nonrandom } A \text { and } B,
$$

where the term with $A$ is due to the short-range interactions, and the term with $B$ contains the effects of the long-range interactions allowed here. In $d \leqq 2$ dimensions a contradiction is avoided only if $M=0$. 
For the $O(N)$ systems with a continuous symmetry, the first term on the right side of (1.3) may be replaced by Const. $L^{d-2}$. That raises the marginal dimension to $d=4$.

Not all the information contained in (1.2) on the limiting distribution of $\tilde{G}_{A}$ is essential for the main result, and the role of (1.2) can also be fulfilled by a suitable lower bound on the moment generating function, $\operatorname{Av}\left(\exp \left(t \tilde{G}_{\Lambda} / \sqrt{|\Lambda|}\right)\right)$. A general bound which is useful for such a purpose is given a self-contained derivation in Appendix II.

The paper is organized as follows. The models discussed here are introduced in Sects. 2 and 3 -the latter containing the general formulation, and the main results are stated in Sect. 4. Section 5 is devoted to the definition of the fluctuating quantity $\tilde{G}_{\boldsymbol{A}}$. In order to guarantee the desired properties, we have to address a basic issue (which in the case of the Random-Field Ising Model is easy to handle) concerning Gibbs states of random systems. The construction of $G_{A}$ is based on an auxiliary result - whose proof is relegated to Appendix I. In Sect. 6 we prove the main Theorem, by establishing (1.2) and (1.3). For the proof of (1.2) we apply the Martingale Central Limit Theorem which is quoted in Appendix II (next to the complete proof of the broader lower bound on the moment generating function). The rounding effect for systems with a continuous symmetry in $d \leqq 4$ dimensions is derived in Sect. 7, whose main content is the sharpening of (1.3) by methods related to proofs of the Mermin-Wagner phenomenon.

\section{Examples: Random-field, Random-bond, and Spin Glass Models}

To make the general notation transparent, let us start by listing some examples. In all cases, we consider systems of bounded spin variables, $\sigma=\left\{\sigma_{x}\right\}$, located on a $d$-dimensional lattice, say $\mathbb{Z}^{d}$. The interaction is a sum of a translation-invariant, non-random term and a fluctuating term with quenched disorder-represented here by a collection of independent random variables $\left\{\eta_{x}\right\}$ with a translation invariant distribution. We denote by $v(d \eta)$ both the product measure for the infinite array $\eta=\left\{\eta_{x}\right\}$, and the individual probability distributions. The latter are assumed to satisfy:

$$
\operatorname{Av}\left(\eta_{x}\right)=0, \quad \operatorname{Av}\left(\eta_{x}^{2}\right)=1 \text { and } \operatorname{Av}\left(e^{s \eta_{x}}\right)<\infty \text { for all real } s,
$$

where $\operatorname{Av}(f)=\int f v(d \eta)(\equiv E(f))$. [The probabilistic notation $E(\cdot)$ will be used here in conjunction with the conditioning on partial information about $\eta$ (sub $\sigma$-algebras) and $\operatorname{Av}(\cdot)$ is used, as in some of the literature, to distinguish the averages over $\eta$ from the thermal averages over $\sigma$.]

i) Random Field (RF) Models [12]. The spins are $N$-dimensional vectors and the interaction is:

$$
H(\sigma)=-\frac{1}{2} \sum_{x, y} J_{x-y} \sigma_{x} \cdot \sigma_{y}-\sum_{x}\left(h+\varepsilon \eta_{x}\right) \cdot \sigma_{x}
$$

This Hamiltonian may include both short-range and long-range interactions, with $J_{x-y} \leqq$ const. $/|x-y|^{\alpha}(\alpha>d)$. Examples of particular interest include: 
a. Ferromagnetic Ising spin models (RFIM), for which: $N=1, \sigma_{x}$ take the values \pm 1 , and $J_{z} \geqq 0$ (that condition is not relevant for the main result, though it does play a role in the translation of the lack of discontinuity in the magnetization to a statement on the uniqueness of the Gibbs state).

b. $O(N)$ models $(N=2$ for the plane-rotor, and $N=3$ for the Heisenberg model) with a rotation-invariant a priori distribution, $\lambda\left(d \underline{\sigma}_{x}\right)$, of bounded support. In that case the dot in (2.2) represents a scalar product.

c. One dimensional long-range models with interactions decaying at a sufficiently slow rate so that the non-random model exhibits a phase transition. That requires $\alpha \leqq 2$, with a strict inequality for the continuous case.

At $\varepsilon=0$ (no randomness) the above models exhibit first-order phase transitions manifested in the discontinuity of the magnetization as a function of $h$ at $h=0$ (in dimensions $d>1$ for case a, and $d>2$ for case b). These discontinuities occur in the thermodynamic variable conjugate to the magnetic field-which is the randomized parameter in (2.2). Thus, the general result proven here shows that in $d \leqq 2$ dimensions, and for $\alpha>3 d / 2$, the discontinuity vanishes when $\varepsilon \neq 0$.

Remarks. 1. If $v(d \eta)$ is not symmetric, then for $\varepsilon \neq 0, h=0$ is no longer a natural location of a possible phase transition (which it is for $\varepsilon=0$ ). Hence, to correctly express the rounding effect of randomness one should consider it at all $h$-as is done here.

2. For RFIM, $M=0$ (the continuity of the thermodynamic magnetization) has the stronger implication that the Gibbs state is unique for almost every realization of $\left\{\eta_{x}\right\}$, at the corresponding values of $(T, h, \varepsilon)$ - see discussion below.

3. To correct a statement made in Ref. [3], we wish to emphasize that the proof of the rounding effect for systems with the continuous symmetry hinges (in dimensions $2<d \leqq 4$ ) on $v(d \eta)$ having the full rotational invariance. (The astonishment expressed by D. Fisher and T. Spencer was justified.)

ii) Random-Bond (RB) $q$-State Potts Models. Here $\sigma_{x}$ take values in $\{1, \ldots, q\}$ and the Hamiltonian is

$$
H(\sigma)=-\frac{1}{2} \sum_{x, y}\left(J_{x-y}+\varepsilon_{x-y} \eta_{x, y}\right) \delta_{\sigma_{x}, \sigma_{y}}
$$

(another RB example is presented in ref. [3]). The distribution of the random parameters $\{\eta\}$ is assumed to be translation-invariant. That means that the $\eta$ 's form a number of classes - one for each value of $z=x-y$, with members of each class being identically distributed and sharing a common strength parameter $\varepsilon_{z}$. In the general notation introduced below we index such classes by $\alpha$.

In addition to the phase transition manifested by spontaneous magnetization, Potts models, with $q$ large enough, exhibit first-order phase transitions with positive latent heat - which is the discontinuity in $\beta$ of the energy density (the variable conjugate to $\beta$ ). As emphasized in the introduction, the random parameters present in (2.3) result (in $d \leqq 2$ dimensions) in the rounding of the latter discontinuity, without eliminating the former.

iii) The Edwards-Anderson Spin Glass (SG) Model, which is a system of Ising 
spins with the Hamiltonian

$$
H(\sigma)=-\frac{1}{2} \sum_{|x-y|=1} \eta_{x, y} \sigma_{x} \sigma_{y}
$$

to which one may add the magnetic field terms. Here the inverse temperature $(\beta)$ is akin to the $\varepsilon$ parameter, since it is the multiplier of the random $\{\eta\}$ - whose distribution is typically assumed to be symmetric. The challenging questions which are presented by this model are discussed (from different perspectives) in refs. [13]. Their thermodynamic manifestations go beyond the specific question of the differentiability of the free energy in $\beta$ which the present work addresses. (For symmetric distributions the differentiability is implied already by the gauge symmetry.) Possible directions for some relevant extensions of our analysis are mentioned in Sect. 4b.

In the next section we present a general formulation of the systems to which our discussion applies. Readers interested mainly in the RFIM may skip to Sect. 4-after a cursory look at the notation presented in Eq. (3.1).

\section{General Systems with Quenched Randomness}

a. The Setup. A statistical mechanical model with quenched randomness is described here by specifying: 1) The state space, $S$, of the single "spin" variables with a finite a priori measure $\lambda(d \sigma)$. 2) The array (lattice) $\mathscr{L}$. (The spin configurations on $\mathscr{L}$ form the "configuration space" $\Omega \equiv S^{\mathscr{L}}$.) 3) The interaction $H(\sigma)$, which contains a translation-invariant part whose parameters (such as $h$ above) affect bulk terms, and also a sum of local terms with varying coefficients. The latter are taken to be random variables, $\{\eta\}$, and the final ingredient of the model is: 4) the distribution law of the random parameters, $v(d\{\eta\})$.

The equilibrium properties of the model concern the free energy, $F$, and the infinite-volume Gibbs states (of the variables $\sigma$ ), corresponding to "typical" configurations of the random parameters $\{\eta\}$. We assume familiarity with these basic concepts, at least in the context of nonrandom, translation-invariant, systems.

In this work we address systems of the above type with the following features.

1. The single spin state space is endowed with a metrizable topology, in which it is compact [see comment below], and thus also separable.

2. $\mathscr{L}$ is a homogeneous lattice, which (to avoid irrelevant notational issues) we just take to be $\mathbb{Z}^{d}$.

3. The Hamiltonian is of the form

$$
H(\sigma)=H_{0}(\sigma)+\sum_{\alpha} \sum_{x}\left(h_{\alpha}+\varepsilon_{\alpha} \eta_{\alpha, x}\right) \cdot g_{\alpha}\left(T_{-x} \sigma\right) .
$$

$H_{0}(\sigma)$ is a translation-invariant interaction, which for specified $\sigma_{\Lambda^{c}}$ depends continuously on $\sigma_{\Lambda}$ (for finite $\Lambda \subset \mathscr{L}$ ). Added to it are random terms in families indexed by $\alpha . T_{x}$ are lattice translations (not to be confused with the temperature $T)$, with which we shall also write

$$
g_{\alpha, x}(\sigma) \equiv g_{a}\left(T_{-x} \sigma\right) .
$$


The index $\alpha$ runs over a collection of finite subsets of the lattice containing the origin, $0 \in \alpha \subset \mathbb{Z}^{d}$, and $g_{\alpha}$ are local, continuous, functions depending on the spins in the set $\alpha .\left\{g_{\alpha}\right\}$ and $\left\{\eta_{a, x}\right\}$ are normalized so that

$$
\sup \left|g_{\alpha}(\sigma)\right| \leqq 1, \quad \text { and } \operatorname{Av}\left(\eta_{\alpha, x}^{2}\right)=1 .
$$

The effective scale of the randomness is set, therefore, by $\left\{\varepsilon_{\alpha}\right\}$.

4. The random variables $\eta_{\alpha, x}$ are jointly independent, with an identical distribution within each $\alpha$-class, satisfying the conditions (2.1).

The index $\alpha$ allows, for example, to include pair interactions of various ranges (as seen in (2.3)-for which $\alpha=\{0, y-x\}$ ), and other multi-spin terms. $\left\{h_{\alpha}\right\}$ are homogeneous coefficients coupled to the same terms as the random $\left\{\eta_{\alpha, x}\right\}$. For interactions of unbounded range some further assumptions are made on the rate of their decay. To avoid encumbering the discussion of the models of prime interest, the decay condition is stated in the second part of this section.

Remark. The main restriction in the assumptions concerning the topological properties of $S$ and the continuity of the interaction is that the spins are bounded. The rest may often be met by a simple adjustment of the topology of $S$. We expect extensions to unbounded spins to be possible, subject to the availability of suitable bounds on the Gibbs states - such as discussed in ref. $[15,26]$.

Bulk properties of random systems are related to the free energy, $F$, which is derived from the finite-volume partition functions $Z_{A}=\int \lambda\left(d \sigma_{A}\right) \exp \left(-\beta H_{A}\right)$. For $T=0$ we interpret the quantity $(-T /|\Lambda|) \ln Z_{\Lambda}(T,\{h\},\{\varepsilon\},\{\eta\})$ as the essential minimum of the finite-volume energy (this makes it continuous in $T$ ). The following proposition summarizes some basically known facts [16].

Proposition 3.1. For each system of the above type, there is a full measure set $\mathscr{N}$ of random couplings $\eta=\left\{\eta_{\alpha, x}\right\}$ (with $v(\mathcal{N})=1$ ) and a function $F(T,\{h\},\{\varepsilon\}$ ) (the free energy) which does not depend on $\eta$, such that:

i) For all $\eta \in \mathcal{N}$ the finite-volume free energy densities converge in the thermodynamic limit to $F$, at all $(T \geqq 0,\{h\},\{\varepsilon\})$,

$$
\lim _{\substack{\Lambda=I-L, L]^{d} \\ L \rightarrow \infty}}-\frac{T}{|\Lambda|} \ln Z_{\Lambda}(T,\{h\},\{\varepsilon\},\{\eta\})=F(T,\{H\},\{\varepsilon\}) .
$$

ii) $F(\cdot)$ is concave in $T$ and $\{h\}$, and continuous at $T=0$-where it equals the ground state energy density for "typical" $\eta$.

iii) If $\eta \in \mathscr{N}$ and $\rho_{\eta}(\cdot)$ is a Gibbs measure for the corresponding interaction, at some $(T>0,\{h\},\{\varepsilon\})$, then

$$
\operatorname{LIM}_{L \rightarrow \infty} \frac{1}{|\Lambda|} \sum_{x \in \Lambda} \rho_{\eta}\left(g_{a, x}\right) \subset\left[\frac{\partial}{\partial h_{\alpha}-0} F(T,\{h\},\{\varepsilon\}), \frac{\partial}{\partial h_{\alpha}+0} F(T,\{h\},\{\varepsilon\})\right],
$$

where LIM is the set of accumulation points of the given sequence. For $T=0$, the same is true with the sum on the left side replaced by $\sum_{x \in \Lambda} g_{\alpha, x}\left(\sigma_{\eta}\right)$, with $\sigma_{\eta}$ any ground state configuration (i.e. one whose energy can not be lowered by changing a finite number of spins). 
By its concavity, $F(\cdot)$ has directional derivatives - as those seen in (3.5). If $F$ is differentiable in $h_{\alpha}$ then, by (3.5), all the Gibbs measures agree on the mean density of $g_{\alpha, x}$. If not, then the system exhibits a first-order phase transition. The corresponding order parameter (defined for $T \geqq 0$ ) is

$$
M_{\alpha}(T,\{h\},\{\varepsilon\})=\frac{1}{2}\left[\frac{\partial}{\partial\left(h_{\alpha}+0\right)}-\frac{\partial}{\partial\left(h_{\alpha}-0\right)}\right] F(T,\{h\},\{\varepsilon\}) .
$$

Equations (3.4) and (3.5) are the random-coupling version of the well known principle for homogeneous systems, with adjustments based on generally known ideas.

Outline of the proof. To derive (3.4) it is convenient to first peel off the contribution from large $\eta,\left|\eta_{\alpha, x}\right| \geqq K$, which (for all $T$ and $h$ ) is bounded by $\frac{1}{|\Lambda|} \sum_{T_{x} \alpha \in}\left|\eta_{\alpha, x}\right| I\left[\left|\eta_{\alpha, x}\right| \geqq K\right]$. By the law of large numbers, and the integrability of $|\eta|$, there is a full measure set, $\mathcal{N}_{0}$, of $\eta$ for which the contribution of this term to the limit is controllable - in the sense of being bounded by some " $\varepsilon(K)$ " $(\rightarrow 0)$. To analyze the main term, we partition the lattice into large congruent blocks, and remove from the interaction the terms coupling distinct regions. The free energy is then decomposed into a sum of independent intra-block terms, and a remainder which is uniformly bounded by $K(a|\partial B|+b \sqrt{|B|}) /|B|$ (B being the elementary block, and the constant $b$ estimating the contribution of the long-range interactions discussed below). The existence of the limit for a given $(T,\{h\},\{\varepsilon\})$ follows by another application of the strong law of large numbers. It follows that there is a full-measure set $\mathscr{N} \subset \mathscr{N}_{0}$ for which the limit exists at a dense (countable) set of values of $(T,\{h\},\{\varepsilon\})$. By the equicontinuity of the main term (which satisfies a Lipschitz condition), and the uniform bounds on the remainders, the convergence extends to all $(T \geqq 0,\{h\},\{\varepsilon\})$, for each $n \in \mathscr{N}$.

For the proof of (3.5) at $T>0$, one may focus on

$$
\begin{aligned}
& \frac{-T}{|\Lambda|} \ln \rho_{\eta}\left(\exp \left[-\beta s \sum_{T_{x} \alpha \subset \Lambda} g_{\alpha, x}\right]\right) \\
& \quad=\frac{-T}{|\Lambda|} \ln \left[Z_{\Lambda}\left(T,\left\{h+s \delta_{\alpha}\right\},\{\varepsilon\}\right) / Z_{\Lambda}(T,\{h\},\{\varepsilon\})\right]+\frac{1}{|\Lambda|} O\left(V_{\partial \Lambda}\right),
\end{aligned}
$$

with $\delta_{\alpha}$ a vector with 1 at the $\alpha$-coordinate and 0 elsewhere, and $V_{\partial \Lambda}$ the boundary term defined in the second part of this section. It is easy to see that for every $\eta \in \mathscr{N}_{0}$ the latter's contribution in (3.7) vanishes, as $L \rightarrow \infty$. For $\eta \in \mathscr{N}$ the above functions of $s$ converge to $F\left(T,\left\{h+s \delta_{\alpha}\right\},\{\varepsilon\}\right)-F(T,\{h\},\{\varepsilon\})$. Since these functions are concave, standard convexity arguments imply that their derivatives obey (3.5). The $T=0$ case is proven by a similar argument, applied to the functions

$$
\begin{aligned}
& \Psi_{\sigma_{\eta} ; \Lambda}(s) \\
& =\frac{1}{|\Lambda|} \inf \left\{H_{\Lambda}\left(\sigma^{\prime}\right)-H_{\Lambda}\left(\sigma_{\eta}\right)+s \sum_{T_{x} \alpha \subset \Lambda} g_{\alpha, x}\left(\sigma^{\prime}\right) \mid \sigma^{\prime} \text { differs only locally from } \sigma_{\eta}\right\} .
\end{aligned}
$$


A particular implication of (3.5) is that if one has a family of $\eta$-dependent Gibbs states, $\rho_{\eta}(-)$, which are weak* measurable in $\eta$ (in the sense that $\rho_{\eta}(f)$ are measurable), and such that $\operatorname{Av}\left(\rho_{\eta}\left(g_{\alpha, x}\right)\right)$ is translation invariant (in $\left.x\right)$, then:

$$
\int v(d \eta) \rho_{\eta}\left(g_{\alpha, 0}\right) \in\left[\frac{\partial}{\partial h-0} F(T, h, \varepsilon), \frac{\partial}{\partial h+0} F(T, h, \varepsilon)\right] .
$$

b. Systems with Long-Range Interactions. The basic restriction for an argument based on (1.2) and (1.3), is that for every cube $\Lambda$ the interaction across the boundary, $V_{\partial \Lambda}$, be of the order of $\sqrt{|\Lambda|}$. That condition requires the dimension to be $d \leqq 2$ (in order to have $\sqrt{|\Lambda|} \leqq$ Const. $|\partial \Lambda|$ ), however it does not restrict the interaction to be of finite range. If the long-range part consists only of the terms $J_{x, y} \sigma_{x} \cdot \sigma_{y}$ then it suffices to require

$$
\left|J_{x, y}\right| \leqq \text { Const. }|x-y|^{-(3 d / 2+\delta)}, \text { for some } \delta>0 .
$$

For the stronger result obtained for systems with continuous symmetry (and $d \leqq 4$ ), the basic condition is somewhat different, however the resulting restriction on $\left\{J_{x, y}\right\}$, given in (4.6), is very close to (3.10).

In the more general statement we refer by $V_{\partial \Lambda}(\sigma)$ to a function of $\sigma$ such that $H-V_{\partial \Lambda}$ decomposes into a sum of two non-interacting terms:

$$
H-V_{\partial \Lambda}=H_{\Lambda}\left(\sigma_{\Lambda}\right)+H_{\Lambda^{c}}\left(\sigma_{\Lambda^{c}}\right) \text {. }
$$

The definition $V_{\partial \Lambda}$ is not unique, since the given interaction $H$ may have more than one representation as a sum of local terms, and the conditions stated below should be read as satisfied if they are met for some such choice. $V_{0, \partial \Lambda}$ is the corresponding quantity for the nonrandom interaction $H_{0}$.

Our assumptions on the Hamiltonian (3.1) are that for every square region $\Lambda=[-L, L]^{d}(d=1,2)$ :

a) $V_{0, \partial \Lambda}$ and $H_{0, \Lambda}$ depend continuously on $\sigma$, and:

where $\|V\|=\sup |V|$.

$$
\left\|V_{0, \partial \Lambda}\right\| \leqq \text { Const. } \sqrt{|\Lambda|},
$$

b) $g_{\alpha}(\sigma)$ are continuous, with $\left\|g_{\alpha}\right\| \leqq 1$ (as stated in (3.3)), and

$$
\sum_{T_{x} \alpha \in \mathscr{D}(\Lambda)}\left(\left|\varepsilon_{\alpha}\right|+\left|h_{\alpha}\right|\right) \leqq \text { Const. } \sqrt{|\Lambda|},
$$

where $\mathscr{D}(\Lambda)=\left\{A \subset \mathscr{L} \mid A \cap \Lambda \neq \varnothing\right.$ and $\left.A \cap \Lambda^{c} \neq \varnothing\right\}$.

Together, the assumption a) and b) imply:

$$
E\left(\left\|V_{\partial \Lambda}\right\|\right) \leqq \text { Const. } \sqrt{|\Lambda|} .
$$

We shall now translate the above conditions to a frequently used terminology. A standard form for a translation-invariant interaction $H_{0}$ is (see [17])

$$
H_{0}(\sigma)=\sum_{A} \Phi_{A}\left(\sigma_{A}\right)
$$

where $A$ ranges over finite subsets of $\mathbb{Z}^{d}$, and $\Phi_{A}$ satisfy the natural translationinvariance condition. Pair interactions are described by $\Phi_{\{x, y\}}\left(\sigma_{x}, \sigma_{y}\right)$. In this 
representation, our assumptions are that $\Phi_{A}$ are continuous (in $\sigma_{A}$ ) and meet the criterion spelled in the next proposition.

Proposition 3.2. In dimensions $d \leqq 2$ :

a) $A$ sufficient condition for the interaction (3.14), to satisfy (3.12a) is

$$
\sum_{\substack{A \ni 0 \\ \operatorname{diam} A \leqq L}} \operatorname{diam}(A) \frac{|\partial A|}{|A|}\left\|\Phi_{A}\right\| \leqq \text { Const. } L^{(2-d) / 2} .
$$

For a pair interaction (3.14), a sufficient condition is

$$
\left\|\Phi_{\{x, y\}}\right\| \leqq \text { Const. }|x-y|^{-3 d / 2} /\left\{1+I[d=2] \ln ^{2}(1+|x-y|)\right\} .
$$

b) In order for (3.12b) to hold it suffices that

$$
\sum_{\substack{\alpha \ni 0 \\ \operatorname{diam}(\alpha) \leqq L}} \operatorname{diam}(\alpha) \frac{|\partial \alpha|}{|\alpha|}\left(\left|\varepsilon_{\alpha}\right|+\left|h_{\alpha}\right|\right) \leqq \text { Const. } L^{(2-d) / 2} .
$$

Proof. a) With the natural choice for $V_{0, \partial \Lambda}$,

$$
\left\|V_{0, \partial \Lambda}\right\| \leqq \sum_{A \in \mathscr{D}(\Lambda)}\left\|\Phi_{A}\right\|
$$

In this sum the contribution from $A$ with diameter smaller than $L$ is at most

$$
\sum_{\substack{A \ni 0 \\ \operatorname{diam}(A) \leqq L}} 2 d L^{d-1} \frac{\operatorname{diam}(A)}{|A|}\left\|\Phi_{A}\right\| \leqq \text { Const. } L^{d / 2}
$$

(where use was made of (3.15)), while the contribution from the remaining $A$ is bounded by

$$
\sum_{\substack{A \ni 0 \\ \operatorname{diam}(A) \geqq L}} L^{d} \frac{|\partial A|}{|A|}\left\|\Phi_{A}\right\| \leqq L^{d-1} \sum_{\substack{A \ni 0 \\ \operatorname{diam}(A) \geqq L}} \operatorname{diam}(A) \frac{|\partial A|}{|A|}\left\|\Phi_{A}\right\| \leqq \text { Const } L^{d / 2}
$$

Hence (3.15). For pair interactions the latter is implied by (3.16). Assertion b) follows by an analogous estimate.

\section{Statement of the Main Results}

Following is our main result, in the setup described in Sect. 3.

\section{a. The Thermodynamic Statement}

Theorem 4.1. In a $d \leqq 2$ dimensional system of the type described in Sect. 3, with independent variables $\eta$ (providing the quenched disorder), and the Hamiltonian (3.1) satisfying the decay conditions (3.12)), for any $\{h\}$ and $T \geqq 0$ :

$$
\varepsilon_{\alpha} \neq 0 \Rightarrow M_{\alpha}(T,\{h\},\{\varepsilon\})=0,
$$

provided $v\left(d \eta_{\alpha}\right)$ is absolutely continuous with respect to the Lebesgue measure. For $T>0$ (4.1) holds under the weaker assumption that $v$ has no isolated point masses. 
In case of the Ising and Potts ferromagnetic RF and RB models, no continuity assumption on the random fields'/couplings' distribution is required for (4.1) at $T>0$.

The proof of the theorem is given in Sect. 6. In part c. of this section we present a result which goes further for systems with continuous symmetries.

b. Consequences for the Gibbs States. Proposition 3.1 shows that whenever $M_{\alpha}=0$, the translation average of $\rho_{\eta}\left(g_{a, x}\right)$ is restricted, for Gibbs measures of "typical" $\eta$, to a single value $\left(\partial F / \partial h_{\alpha}\right)$. (One should note that there is a difference between such statements with exclusions of sets of zero measure applied separately at each $(T, h)$, and the stronger statements with a uniform restriction to a full measure set, like $\mathscr{N}$ of Proposition 3.1.) Hence we have the following result.

Corollary 4.2. In a $d \leqq 2$ dimensional system as in Proposition 4.1, the following is true for all $\eta \in \mathscr{N}$ (with $\mathcal{N}$ the full measure set described in Proposition 3.1). If $\rho_{\eta}(\cdot)$ and $\rho_{\eta}^{\prime}(\cdot)$ are two Gibbs states for $H_{\eta}$, at common values of $(T>0,\{h\},\{\varepsilon\})$ with $\varepsilon_{\alpha} \neq 0$, then:

$$
\lim _{\substack{\Lambda=[-L, L]^{d} \\ L \rightarrow \infty}} \frac{1}{|\Lambda|} \sum_{T_{x} \alpha \subset \Lambda}\left[\rho_{\eta}\left(g_{\alpha, x}\right)-\rho_{\eta}^{\prime}\left(g_{\alpha, x}\right)\right]=0 .
$$

Similarly, for any two ground state configurations of $H_{\eta}\left(\sigma_{\eta}\right.$ and $\left.\sigma_{\eta}^{\prime}\right)$,

$$
\lim _{\substack{\Lambda=[-L, L]^{d} \\ L \rightarrow \infty}} \frac{1}{|\Lambda|} \sum_{T_{x} \alpha \subset \Lambda}\left[g_{\alpha, x}\left(\sigma_{\eta}\right)-g_{\alpha, x}\left(\sigma_{\eta}^{\prime}\right)\right]=0,
$$

provided $v$ satisfies the stronger $(T=0)$ condition of Proposition 4.1, i.e. is absolutely continuous.

More can be deduced for the ferromagnetic Ising, and Potts, random-field and random-bond models.

Corollary 4.3. For a $d \leqq 2$ dimensional ferromagnetic Ising model, satisfying the conditions specified in Theorem 4.1, at each $\{T, h, \varepsilon \neq 0\}$ the Gibbs state is unique for $v$-a.e. set of random couplings $\eta$.

Sketch of the Argument. In RFIM the Fortuin-Kasteleyn-Ginibre (FKG) inequality [18] is satisfied for an arbitrary field configuration $\left\{\eta_{x}\right\}$. The ensuing domination arguments show that in the partial order of the Gibbs measures by the expectation values of "monotone" functions there is a pair forming the "lowest" and the "highest" states (constructed by taking the infinite-volume limit of the system with uniform -1 , or +1 , boundary conditions) for which the differences in (4.2) are all nonnegative. Under these conditions, (4.2) implies that if $M\{T, h, \varepsilon\}=0$ then for "typical" random couplings the Gibbs state is unique.

One could also ask whether it is true that for each "typical" $\eta$ there are no exceptional values of $\{T, h\}$ (which would have to form a random set, dense in some nonrandom region) at which there is more than one state. Equation (4.2) does not quite rule that out, though it does imply that differences can occur at no more than zero fractions of the volume. In this sense, we can say that for "typical" $\eta$ (e.g. all elements of $\mathcal{N}$ ) for no value of $\{T, h, \varepsilon \neq 0\}$ is there a bulk first-order phase transition. 
Thus, the situation for $\varepsilon>0$ is drastically different from the case $\varepsilon=0$. The continuity restriction on $v(d \eta)$ is not totally superfluous at $T=0$ (though it could be eased), since in the RFIM if $v(d \eta)$ has a discrete component then at least for certain values of $\varepsilon>0$ the ground state is typically nonunique - with a macroscopic degeneracy. On the other hand, for $T>0$ we expect the restriction to be unnecessary even in the general case.

Remark. For the spin glass models with symmetric $v(d \eta)$, the general theorem presented here yields no new information even for $d=2$ dimensions, since its conclusion holds by the model's "gauge symmetry." However, certain questions posed by this, and other models (e.g. the random bond antiferromagnetic model in external field [19]), would be resolved by an improved version of (4.3), with the difference $\left[g_{\alpha, x}\left(\sigma_{\eta}\right)-g_{\alpha, x}\left(\sigma_{\eta}^{\prime}\right)\right]$ replaced by its absolute value. The issue is related to some points which were raised in the comprehensive theory proposed by Fisher and Huse for spin glass models. Its elucidation calls for further refinement of the methods presented here and in ref. [14]. (We wish to thank D. Fisher and J. Bricmont for stimulating discussions of the relation of this work to the above mentioned models.)

c. Systems with Continuous Symmetry. In the presence of continuous symmetry, the rounding effect of quenched disorder extends up to the higher dimension $d=4$. We consider here systems of $N$-dimensional spins, which are invariant (with the random interaction terms only stochastically invariant) under the action of one of the closed connected subgroups of $O(N)$, denoted by $\mathscr{G}$. Because of the paramount role of the symmetry, we adopt here - and in Sect. 7 , the vector notation $(\sigma, \underline{h}, \underline{\eta})$. In a brief form, the statement we prove, for $d \leqq 4$ and under the suitable assumptions, is that at $\underline{h}=0$ the free energy has vanishing directional derivatives in all the directions within the orthogonal complement of the subspace of the invariant vectors: $I_{\mathscr{G}}=\left\{\underline{u} \in \mathbb{R}^{N} \mid R \underline{u}=\underline{u}\right.$ for all $\left.R \in \mathscr{G}\right\}$. For the " $O(N)$ case" of $(2.2)$ that translates to differentiability at $\underline{h}=\underline{0}$, since $I_{\mathscr{G}}^{\perp}=\mathbb{R}^{N}$.

For systems with pair interactions we write the Hamiltonian as:

$$
H=\sum_{x, y} J_{x-y} \Psi_{x-y}\left(\underline{\sigma}_{x}, \underline{\sigma}_{y}\right)-\sum_{x}\left(\underline{h}+\varepsilon \underline{\eta}_{x}\right) \cdot \underline{\sigma}_{x},
$$

$\left\|\Psi_{x-y}\right\| \leqq 1$; and make the additional symmetry assumptions, that for all $R \in \mathscr{G}$ :

i) the a priori measure is $R$-invariant: $\lambda\left(d \underline{\sigma}_{x}\right)=\lambda\left(d R \underline{\sigma}_{x}\right)$,

ii) $\Psi_{x-y}\left(R \underline{\sigma}_{x}, R \underline{\sigma}_{y}\right)=\Psi_{x-y}\left(\underline{\sigma}_{x}, \underline{\sigma}_{y}\right)$, for all $\left\{x, y, \underline{\sigma}_{x}, \underline{\sigma}_{y}\right\}$,

iii) $v(d \underline{\eta})=v(d R \underline{\eta})$, though for each realization of the random coefficients the interaction is of course not $\mathscr{G}$-invariant.

Furthermore, we make the smoothness assumptions

iv) $\Psi_{x-y}\left(R \underline{\sigma}_{x}, \underline{\sigma}_{y}\right)$ is twice differentiable in $R$, along $\mathscr{G}$-with respect to the Lie group structure inherited by $\mathscr{G}$ from $O(N)$. It is normalized so that for any one-parameter subgroup $\left\{R_{\theta}\right\} \subset \mathscr{G}$, with the natural parametrization (which in the vicinity of the identity is given by the maximal angle by which $R$ rotates a vector),

$$
\sup _{\underline{\sigma}, \underline{\sigma}^{\prime}} \sup _{\theta}\left|\frac{\partial^{2}}{\partial \theta^{2}} \Psi_{x-y}\left(\underline{\sigma}, R_{\theta} \underline{\sigma}^{\prime}\right)\right| \leqq 1 .
$$


The interaction may be of unbounded range, and our result applies as long as: v) $\sum_{z \in Z^{d}:|z| \leqq L}\left|J_{z}\right||z|^{2} \leqq$ Const. $L^{(4-d) / 2}$,

for which it suffices (if $d \leqq 4$ ) that

$$
\left|J_{x-y}\right| \leqq \text { Const. }|x-y|^{-3 d / 2} /\left\{1+I[d=4] \ln ^{2}(1+|x-y|)\right\} \text {. }
$$

Even though for each realization of $\eta$ the system's interaction is not symmetric under $\mathscr{G}$, such a symmetry exists at the bulk level (by the symmetry of the probability distribution of $\eta$ and its ergodicity under translations). In particular, it is easy to see (e.g. from the first part of Proposition 3.1) that the free energy $F(T, \underline{h}, \varepsilon)$ is $\mathscr{G}$-invariant:

$$
F(T, R \underline{h}, \varepsilon)=F(T, \underline{h}, \varepsilon), \text { for all } R \in \mathscr{G} .
$$

It makes sense, therefore, to say that a symmetry breaking occurs if an interaction, with a field configuration which is not "a typical", admits at $\underline{h}=\underline{0}$ a Gibbs state $\rho_{\eta}(\cdot)$ whose bulk magnetization is not an invariant vector or more precisely if its set of accumulation points does not satisfy:

$$
\operatorname{LIM}_{L \rightarrow \infty} \frac{1}{|\Lambda|} \sum_{x \in \Lambda} \rho_{\eta}\left(\underline{\sigma}_{x}\right) \subset I_{y}
$$

(in the notation of (3.5)). That possibility is ruled out if the directional derivatives of $F(T, \underline{h}, \varepsilon), D(\underline{v}) f(h)=\left(d / d s^{+}\right) f(\underline{h}+s \underline{v})$ - which exists by the convexity of $F$ in $\underline{h}$, satisfy:

$$
D(v) F(T, 0, \varepsilon)=0, \text { for all } \underline{v} \in I_{G}^{\perp} .
$$

The reason is that if (4.9) holds then, by (3.5), $\lim _{L \rightarrow \infty} \frac{1}{|\Lambda|} \sum_{x \in \Lambda} \rho_{\eta}\left(\underline{v} \cdot \underline{\sigma}_{x}\right)=0$ for each $\underline{v} \in I_{g}^{\perp}$, which implies (4.8).

By the celebrated Mermin-Wagner theorem, in non-random systems there is no continuous symmetry breaking (under the suitable assumptions) in dimensions $d \leqq 2$. The following result (proven here in Sect. 7) states that quenched randomness inhibits continuous symmetry breaking over a broader range of dimensions.

Theorem 4.4. Assume the system has the properties listed above, with the symmetry group $\mathscr{G}$ being a connected subgroup of $O(N), N \geqq 2$. If the dimension is $d \leqq 4$, and $v(d \eta)$ is absolutely continuous with respect to the $N$-dimensional Lebesgue measure, then for $\varepsilon \neq 0 F(T, h, \varepsilon)$ is differentiable in $\underline{h}$, at $\underline{h}=\underline{0}$ (for all $T \geqq 0)$, in the directions lying in the orthogonal complement of $I_{y}$, i.e. it satisfies (4.9). For $T>0$, the absolute continuity assumption can be replaced by the requirement that $v(d \eta)$ be spherically symmetric, and have no isolated points in its radial distribution [in the sense made explicit in Appendix III, b].

As in clear from the proof (Sect. 7), the theorem has a natural generalization to systems with many-body interactions, the relevant condition being the energy bound of Lemma 7.5. 


\section{The Fluctuating Quantity $G_{A}(\eta)$}

In this section we construct the finite-volume quantity $G_{\Lambda}(\eta)$ on which the argument outlined in (1.2) and (1.3) focuses. Instrumental for the construction is the statistical mechanical manifestation of the thermodynamically defined $M_{\alpha}$, as the discontinuity of the average (over the randomness or over the translations) of the thermal expectation value of the quantity conjugate to $h_{\alpha}$ and $\left\{\eta_{\alpha, x}\right\}$. If $M_{\alpha}(T, h, \varepsilon) \neq 0$ then for typical configurations of the couplings, i.e. for $v$-a.e. $\eta$, the spin system has at least two Gibbs states which differ (by $\left.2 M_{\alpha}\right)$ in the bulk density of $g_{\alpha, x}(\sigma)$. Our $G_{\Lambda}(\eta)$ is, in essense, the difference between the finite-volume free energies of pairs of such states. The basic construction is simplest to present under an auxiliary condition - the existence of $\eta$-covariant states (in the terminology introduced below), which is satisfied in RFIM and in the ferromagnetic random bond Potts models. Before turning to the generalizations, and to the interesting questions which arise in that context, let us present $G_{\Lambda}(\eta)$ in the simplest case.

In the case of RFIM, our purpose could be served by the quantity

$$
\mathbb{G}_{\Lambda}\left(\eta_{\Lambda}\right)=T \ln Z_{\Lambda,+}(T, h,\{\varepsilon \eta\})-T \ln Z_{\Lambda,-}(T, h,\{\varepsilon \eta\}),
$$

where $Z_{\Lambda, \pm}$ are the partition functions with the standard $( \pm)$ boundary conditions. It is however more convenient-both for RFIM and from the general point of view, to work with a slightly different quantity (which has a built-in translation covariance). Let $\rho_{\eta,+}$ and $\rho_{\eta,-}$ be the Gibbs measures for the random field $\eta$, obtained in the infinite volume limit by means of the above mentioned boundary conditions: $\sigma_{\Lambda^{c}}=1$, and, correspondingly, $\sigma_{\Lambda^{c}}=-1$. We define:

$$
G_{\Lambda}(T, h,\{\varepsilon \eta\})=\frac{T}{2} \ln \rho_{\eta,-}\left(\exp \left(\beta \sum_{x \in \Lambda} \varepsilon \eta_{x} g_{x}\right)\right)-\frac{T}{2} \ln \rho_{\eta,+}\left(\exp \left(\beta \sum_{x \in \Lambda} \varepsilon \eta_{x} g_{x}\right)\right) .
$$

with $\beta \equiv 1 / T$. (One may notice that the terms in the exponents in (5.2) cancel similar terms in the Gibbs factors of the $\rho_{\eta, \pm}$ states. That cancellation is made explicit in (5.12) below.)

The relevant properties of the Ising model's states $\rho_{\eta, \pm}$ are summarized by their " $\eta$-covariance"-in the terminology defined below.

Definition. An $\eta$-covariant state [at the given $(\beta,\{h\},\{\varepsilon\})]$, is a measurable function (in the weak* sense explained in Appendix I) associating a Gibbs measure $\rho_{\eta}(-)$ to each $\eta$ in a set of the full $v$-measure, which changes with $\eta$ in the following way:

i) Under local changes-i.e. for $\Delta \eta$ with a finite number of nonzero terms,

$$
\rho_{\eta}+\Delta \eta(-)=\rho_{\eta}\left(-e^{-\beta \Delta H}\right) / \rho_{\eta}\left(e^{-\beta \Delta H}\right),
$$

where

$$
\Delta H=\sum_{\alpha} \sum_{x} \varepsilon_{\alpha}(\Delta \eta)_{\alpha, x} g_{\alpha, x}
$$

(see (3.1) and (3.2)). 
ii) Under the lattice translations, $T_{x}$,

$$
\rho_{T_{x \eta}}(f)=\rho_{\eta}\left(T_{-x} f\right)
$$

for all observables $f$.

An $\eta$-covariant state which is a limit of $\eta$-covariant states with $h_{\alpha}$ varied over a sequence of values $h_{\alpha}^{(n)} \downarrow h_{\alpha}$ (correspondingly $\left.h_{\alpha}^{(n)} \uparrow h_{\alpha}\right)$, is referred to as an $\alpha$-plus $\left(\alpha_{+}\right)$ state (or an $\alpha$-minus $\left(\alpha_{-}\right)$state).

Speaking somewhat loosely, a sufficient condition for the existence of $\eta$-covariant $\left(\alpha_{ \pm}\right)$states is the availability of some $\eta$-independent and translation-invariant boundary conditions, with which the finite-volume Gibbs states converge, for at least $v$-a.e. $\eta$. For RFIM such a role is played by the above mentioned boundary conditions $\sigma_{\Lambda^{c}} \equiv \pm 1$. For the ferromagnetic random-coupling Potts models an $\eta$-covariant + phase may be constructed by means of the uniformly ordered boundary condition, and a - phase is achieved by means of the free boundary conditions. [We omit the proof of these statements (based on correlation inequalities, notably the FKG inequality [18]), since they are standard and are not essential for our main result.] The question of the general existence of such states poses, however, a hitherto unexpected difficulty (related to some physically interesting issues, concerning the possible structures of the collections of extremal Gibbs states). We bypass the difficulty by moving the discussion one rung up - to the level of probability measures on the space of states. At this level we do find, quite generally, measures with the desired $\eta$-covariance. The relevant existence result is stated in Proposition 5.1 (and proven in Appendix I).

For the simpler case, of systems which possess $\eta$-covariant $\alpha_{ \pm}$phases $\left(\rho_{\eta, \alpha, \pm}\right)$ we define $G_{\Lambda, \alpha}$ as follows:

$$
\begin{aligned}
G_{\Lambda, \alpha}(T,\{h\},\{\varepsilon \eta\})= & \frac{T}{2} \log \rho_{\eta, \alpha,-}\left(\exp \left(\beta \sum_{\substack{x: \\
T_{x} \subset \Lambda}} \varepsilon_{\alpha} \eta_{\alpha, x} g_{\alpha, x}(\sigma)\right)\right) \\
& -\frac{T}{2} \log \rho_{\eta, \alpha,+}\left(\exp \left(\beta \sum_{\substack{x: \\
T_{x}^{\alpha}<\Lambda}} \varepsilon_{\alpha} \eta_{\alpha, x} g_{\alpha, x}(\sigma)\right)\right) .
\end{aligned}
$$

The next proposition lays the grounds for a generalization of this object, which allows our argument to proceed regardless of the existence of $\eta$-covariant states. For the proof of the rounding effect in RFIM (and the other models in the special class described above) the reader may now skip to Proposition 5.2, which presents the relevant properties of $G_{\boldsymbol{A}}$.

Proposition 5.1. For each system of the type described in Sect. 3, at each $(\beta, h,\{\varepsilon\})$ there exists a function $\mu_{\eta}$, defined for $\eta$ in a measurable set $\mathscr{N}$ with $v(\mathscr{N})=1$, whose values are probability measures on the space of states of the spin system, with the following properties:

i) The map $\eta \rightarrow \mu_{\eta}$ is measurable (in a weak* sense-see Appendix I).

ii) For each $\eta \in \mathcal{N}$, the probability measure $\mu_{\eta}(d \rho)$ is supported in the set of Gibbs measures for the Hamiltonian corresponding to $\eta$. 
iii) Under local changes in $\eta$, with $\Delta \eta$ consisting of only a finite number of nonzero terms, $\mu_{\eta}$ is transformed as follows:

$$
\mu_{\eta+\Delta \eta}(d \rho)=\mu_{\eta}\left(d\left(U_{\Delta \eta}^{-1} \rho\right)\right)
$$

where $U_{\Delta \eta}$ is the map taking a state $\rho$ to $U_{\Delta \eta} \rho(-) \equiv \rho\left(-e^{-\beta \Delta H}\right) / \rho\left(e^{-\beta \Delta H}\right)$, with $\Delta H$ defined in (5.4).

iv) $\mu_{\eta}$ is a translation-covariant function of $\eta$, meaning that under the lattice translations

$$
\mu_{T_{x \eta}}(d \rho)=\mu_{\eta}\left(d T_{-x} \rho\right)
$$

with $T_{-x} \rho(f) \equiv \rho\left(T_{x} f\right)$.

v) Furthermore, for any $\alpha$ there is a pair of measures, $\mu_{\eta, \alpha,+}$ and $\mu_{\mu, \alpha,-}$, satisfying

$$
\int v(d \eta) \int \mu_{\eta, \alpha, \pm}(d \rho) \rho\left(g_{\alpha, 0}\right)=\frac{\partial}{\partial h_{\alpha} \pm 0} F(T,\{h\},\{\varepsilon \eta\}) .
$$

The proof of the proposition is given in Appendix I.

Remark. Using the general notion of the baricenter, we associate to each of the above measures, $\mu_{\eta}(d \rho)$, the single $\eta$-dependent state:

$$
\rho_{\eta}^{\mu}(-)=\int \mu_{\eta}(d \rho) \rho(-) \text {. }
$$

The baricenter of $\mu_{\eta}$ is a Gibbs state for the couplings $\eta$ (being a superposition of Gibbs states), which is covariant under translations (i.e. satisfies (5.5)). However, the simple behavior of the measures $\mu_{\eta}$ under local changes in $\eta$ (Eq. (5.7)) does not automatically extend to a simple transformation law for $\rho_{\eta}^{\mu}$. The reason is that $U_{\Delta \eta}$ is not an affine map.

A related observation is that $\rho_{\eta}^{\mu}$ does not necessarily provide the full information on the measure $\mu_{\eta}$-since the support of $\mu_{\eta}$ is not restricted to the set of extremal Gibbs states.

We are now ready to define the fluctuating quantity $G_{\Lambda, \alpha}(\eta)$, which is used in the proof of the vanishing of $M_{\alpha}$ for a particular value of the index $\alpha$ (which will often be suppressed). Starting with a pair of measures $\mu_{\eta,-}(d \rho)$ and $\mu_{\eta,+}(d \rho)$ with the properties i)-v) listed in Proposition 5.1 (in some models the pair is not unique), we define:

$$
\begin{aligned}
G_{\Lambda, \alpha}(\eta) & =\frac{1}{2 \beta} \int\left[\mu_{\eta, \alpha,+}(d \rho)-\mu_{\eta, \alpha,-}(d \rho)\right] \ln \rho\left(\exp \left(+\beta \sum_{\substack{x: 1 \\
T_{x} \alpha \subset \Lambda}} \varepsilon_{\alpha} \eta_{\alpha, x} g_{\alpha, x}\right)\right) \\
& \equiv \frac{1}{2 \beta} \int \mu_{\eta, \alpha,+}\left(d \rho_{+}\right) \mu_{\eta, \alpha,-}\left(d \rho_{-}\right) \ln \left[\frac{\rho_{+}\left(\exp \left(+\beta \sum_{x: T_{x}^{\alpha \subset \Lambda}} \varepsilon_{\alpha} \eta_{\alpha, x} g_{\alpha, x}\right)\right)}{\rho_{-}\left(\exp \left(+\beta \sum_{x: T_{x} \alpha \subset \Lambda} \varepsilon_{\alpha} \eta_{\alpha, x} g_{\alpha, x}\right)\right)}\right] .
\end{aligned}
$$

The signs seen in the exponents in (5.11) are chosen to be the opposite of the signs in the Gibbs factors. An alternative expression for $G_{\Lambda, \alpha}(\eta)$, which better 
manifests its similarity to $\mathbb{G}_{\Lambda}(\eta)$ of $(5.1)$, is

$$
=\frac{1}{2 \beta} \int \mu_{\eta_{\Lambda^{c}, \alpha,+}}\left(d \rho_{+}\right) \mu_{\eta_{\Lambda^{c}, \alpha,-}}\left(d \rho_{-}\right) \ln \left[\frac{\rho_{+}\left(\exp \left(-\beta \sum_{T_{x^{\alpha} \alpha \Lambda}} \varepsilon_{\alpha} \eta_{\alpha, x} g_{\alpha, x}\right)\right)}{\rho_{-}\left(\exp \left(-\beta \sum_{T_{x} \alpha \subset \Lambda} \varepsilon_{\alpha} \eta_{\alpha, x} g_{\alpha, x}\right)\right)}\right] \text {. }
$$

where we apply the convention that $\eta_{\Lambda^{c}}$ is the random field configuration which is zero for $(\alpha, x)$ with $T_{x} \eta \subset \Lambda$, and agrees with $\eta$ in $\Lambda^{c}$ (the complement of $\Lambda$ ). The equivalence of (5.12) with (5.11) is a consequence of (5.7).

Remark. For systems with $\eta$-covariant states, the construction described in Proposition 5.1 can be attained with point measures. In this case, our generalization of $G_{A}$ would be consistent with (5.6).

The following statement summarizes the relevant properties of $G_{\Lambda}(\eta)$.

Proposition 5.2. The quantity defined by (5.11) ( and-when applicable-(5.6)), has the following properties:

i) For $(x, \alpha)$ with $T_{x} \alpha \in \Lambda$,

$$
\frac{\partial G_{\Lambda}}{\partial \eta_{\alpha, x}}=\frac{\varepsilon_{\alpha}}{2}\left[\rho_{\eta,+}\left(g_{\alpha, x}\right)-\rho_{\eta,-}\left(g_{\alpha, x}\right)\right] \equiv \varepsilon_{\alpha} \tau_{\alpha, x},
$$

where the states $\rho_{\eta, \pm}$ are the baricenters of the measures $\mu_{\eta, \pm}$, defined by (5.10).

ii) The averages over $\eta$ yield the order parameter

$$
\operatorname{Av}\left(\frac{\partial G_{\Lambda}(\eta)}{\partial \eta_{\alpha, x}}\right)=\varepsilon_{\alpha} M_{\alpha}(T,\{h\},\{\varepsilon\})
$$

iii) $G_{\Lambda}(\eta)$ obeys the general bound:

$$
\left|G_{\Lambda}(\eta)\right| \leqq 2\left\|V_{\eta ; \partial \Lambda}\right\|,
$$

where $V_{\eta ; \partial \Lambda}(\sigma)$ is the interaction across the boundary of $\Lambda$, discussed in Sect. $3 b$, and the norm $\|\cdot\|$ refers to the supremum over the spins.

Proof. The formula for the derivative is an elementary consequence of the representation (5.12) of $G_{A}$. It should be noted that unlike (5.12), the expression provided for $G_{A}$ by (5.11) depends on $\eta_{A}$ in two places.

ii) follows from the explicit expression (5.13), and the properties (5.7) and (5.8) of the measures $\mu_{\eta, \pm}$.

To prove iii) let us note that $G_{A}$ is the ratio of two expectation values of the same quantity, which is a function of the spins in $\sigma$, taken in a pair of Gibbs states $\rho_{+}$and $\rho_{-}$. These states are of the form $\rho_{ \pm}(\cdot)=\tilde{\rho}_{ \pm}\left(\cdot e^{-\beta V_{\partial \Lambda}}\right) / \tilde{\rho}_{ \pm}\left(e^{-\beta V \lambda \Lambda}\right)$ with $\tilde{\rho}_{ \pm}$a pair of measures with identical restrictions to the region $\Lambda$. That easily implies (5.15).

Our results on the Gaussian behavior (derived in the next section) are formulated for averaged versions of $G_{\boldsymbol{A}}$. For a fixed $\alpha$, we define:

$$
\tilde{G}_{\Lambda, \alpha}=E\left(G_{\Lambda, \alpha} \mid \eta_{\Lambda}\right)-E\left(G_{\Lambda, \alpha}\right)
$$


where $E(\cdot)$ stands for the (conditional) expectation with respect to $v(d \eta)$, and $\eta_{\Lambda}=\left\{\eta_{x, \alpha} \mid T_{x} \alpha \subset \Lambda\right\}$. Note that with this choice $V_{\eta ; \partial \Lambda}(\sigma)$ has no terms involving $\eta_{\Lambda}$. Hence, in $d \leqq 2$ dimensions, (5.15) together with (3.12) imply the uniform bound:

$$
\left|\tilde{G}_{\Lambda, \alpha}\left(\eta_{\Lambda}\right)\right| \leqq A \sqrt{|\Lambda|}
$$

In the following discussion the index $\alpha$ is often omitted, e.g. $\eta_{\alpha, x}$ will be written as $\eta_{x}$.

\section{Proof of the Rounding of First-Order Phase Transitions in $d \leqq 2$ Dimensions}

The proof will follow the strategy outlined in Sect. 1b. Since the free energy bound (1.3) was established in (5.17), it remains to derive the Gaussian behavior stated in (1.2). That is done in the following proposition, which is stated in a notation slightly detached from the immediate application-since the criterion may be of interest also in other setups.

Proposition 6.1. Let $\Gamma_{\Lambda}\left(\eta_{\Lambda}\right)$ be a collection of functions of the I.I.D variables $\left\{\eta_{\alpha, x}\right\}_{x: T_{x} \alpha \Lambda}$ (with the given value of $\alpha$ ), indexed by $\Lambda=[-L, L]^{d} \subset \mathbb{Z}^{d}$. If there exists a translation covariant function $\tau_{\alpha, x}(\eta)$, such that:
i) $\frac{\partial \Gamma_{\Lambda}\left(\eta_{\Lambda}\right)}{\partial \eta_{\alpha x}}=\varepsilon E\left(\tau_{\alpha, x} \mid \eta_{\Lambda}\right)$ for each $x$, with $T_{x} \alpha \subset \Lambda$,
ii) $\operatorname{Av}\left(\tau_{\alpha, x}\right)=M$,
iii) $\left|\tau_{\alpha, x}(\eta)\right| \leqq 1$, and $\left|\frac{\partial \tau_{\alpha, 0}(\eta)}{\partial \eta_{\alpha, 0}}\right| \leqq \varepsilon / T \quad$ (uniformly in $\eta$ )

and, furthermore,

iv) $\operatorname{Av}\left(\Gamma_{\Lambda}\right)=0$,

then the distribution of $\Gamma_{\Lambda}$ has a Gaussian limit-

$$
\Gamma_{\Lambda} / \sqrt{|\Lambda|} \stackrel{\mathscr{D}}{\rightarrow} N\left(0 . b^{2}\right)
$$

with some constant $b$, obeying

$$
\varepsilon \theta_{v}(M, T / \varepsilon) \leqq b \leqq \varepsilon \sqrt{2 \operatorname{Av}\left(|\eta|^{2}\right)}
$$

with the function $\theta_{v}(M, T / \varepsilon)$ defined in Appendix III (Eq. (A.3.2).

In particular, the moment generating function of $\Gamma_{\boldsymbol{\Lambda}}$ satisfies:

$$
\liminf _{\substack{\Lambda=[-L, L]^{d} \\ L \rightarrow \infty}} \operatorname{Av}\left(\exp \left(t \Gamma_{\Lambda} / \sqrt{|\Lambda|}\right)\right) \geqq \exp \left(\frac{t^{2}}{2} \varepsilon^{2} \theta_{v}^{2}(M, T / \varepsilon)\right)
$$

for all real $t$.

If, in addition, $\tau_{0}(\eta)$ is monotone in $\eta_{0}$, then the function $\theta_{v}(\cdot)$ may be replaced in (6.7) by $\gamma_{v}(\cdot)$ (defined in (A.3.2)).

Remarks. The above proposition is proven below by verifying the conditions of a general central limit theorem, stated in Appendix II. The bound (6.7) on the 
moment generating function, which suffices for our main purpose, is stated here explicitly since it requires a somewhat less demanding criterion-which is derived in Appendix II. The main result (Theorem 4.1) is thus given here a completely self-contained proof.

Before turning to the proof of the proposition let us just point out its implication for our main problem.

Proof of Theorem 4.1 (assuming Proposition 6.1). Let $\Gamma_{\Lambda}=\tilde{G}_{\Lambda} \equiv \tilde{G}_{\Lambda, \alpha \cdot}$ It directly follows from Proposition 5.2 that the conditions (6.1)-(6.4) are satisfied, regardless of the dimension $d$. (One may note, however, that in the passage from (5.11) to (6.1) use is being made of the independence of $\eta$-which permits the interchange of the differentiation with respect to $\eta_{x}$ with the partial integration over other $\eta$.) Therefore $\widetilde{G}_{\boldsymbol{A}}$ also satisfies the bound (6.7). On the other hand, (5.17) implies

$$
\operatorname{Av}\left(\exp \left(t \tilde{G}_{\Lambda} / \sqrt{|\Lambda|}\right)\right) \leqq \exp (t \cdot A), \text { for all } t \geqq 0 \text {. }
$$

In $d=2$ dimensions (6.7) is inconsistent with (5.17) unless $\theta_{v}(M, T / \varepsilon)=0$ (andwhen applicable, also $\gamma_{v}(M, T / \varepsilon)=0$, with the contradiction arising at sufficiently high values of $t$. Under the continuity assumptions on $v$, stated in Proposition 4.1, the statements $\theta_{v}(M, T / \varepsilon)=0$ and-if available- $\gamma_{v}(M, T / \varepsilon)=0$, imply $M=0$.

Proposition 6.1 is derived by invoking the general criteria presented in Appendix II. In order to do so, we first establish the relevant conditions. That is the content of the following Lemma 6.2. We start by defining some $\sigma$-algebras which are useful in the martingale representation of $\Gamma_{\boldsymbol{A}}$. For any rectangular region $\Lambda$, let us list the sites $x$ such that $T_{x} \alpha \subset \Lambda$ (e.g. using the lexicographic order of the lattice $\mathbb{Z}^{d}$ ) as $x_{1}, \ldots, x_{|\Lambda|}$. (If $\alpha$ contains more than one point, then the number of such sites differs from $|\Lambda|$ by a term bounded by $|\partial \Lambda| \cdot \operatorname{diam}(\alpha)$. This point is inconsequential, and will be ignored. Furthermore, to keep the notation simple, we refer to the above condition on $x$ simply by $x \in \Lambda$.) We denote by $\mathscr{F}_{\Lambda, k}$ the $\sigma$-algebras generated by $\left\{\eta_{x_{i}} \mid 1 \leqq i \leqq k\right\}$, with $F_{\Lambda} \equiv \mathscr{F}_{\Lambda,|\Lambda|}$. We now decompose $\Gamma_{\Lambda}$ as the following sum of mean-zero, orthogonal, increments

$$
\Gamma_{\Lambda}(\eta)=\sum_{k=1}^{|\Lambda|} Y_{\Lambda, k}(\eta)
$$

with

$$
Y_{\Lambda, k}(\eta)=E\left(\Gamma_{\Lambda} \mid \mathscr{F}_{\Lambda, k}\right)-E\left(\Gamma_{\Lambda} \mid \mathscr{F}_{\Lambda, k-1}\right)
$$

Lemma 6.2. For the rectangular regions $\Lambda=[-L, L]^{d}$, with $L \rightarrow \infty$, the variables $Y_{\Lambda, k}(\eta)$ satisfy:

i) For each $\eta, \quad\left|Y_{\Lambda, k}(\eta)\right| \leqq \varepsilon \beta\left[\left|\eta_{x}\right|+\operatorname{Av}(|\eta|)\right]$.

ii) There is a finite number $b$, satisfying (6.6), such that

$$
\left|\frac{1}{|\Lambda|} \sum_{k=1}^{|\Lambda|} E\left(Y_{\Lambda, k}^{2} \mid \mathscr{F}_{\Lambda, k-1}\right)-b^{2}\right| \underset{L \rightarrow \infty}{\stackrel{d}{\longrightarrow}} 0 .
$$


For models (like RFIM) with $\tau_{0}(\eta)$ monotone in $\eta_{0}$, the function $\theta_{v}(\cdot)$ may be replaced in (6.6) by $\gamma_{v}(\cdot)$.

Proof. For any lattice site $x$, we denote by $\eta_{<x}$ the collection of variables $\left\{\eta_{y} \mid y \in \mathbb{Z}^{d}\right.$, $y<x$ (in the lexicographic order) $\}$ - and by $\eta_{>x}$ and $\eta_{\leqq x}$ the collections defined by the corresponding conventions. The conditional expectation, conditioned on $\eta_{\leqq x}$ may naturally be written (in a notation which we hope is suggestive enough) by means of an integral over $\bar{\eta}_{>x}$, as in the following example:

$$
E\left(\Gamma_{\Lambda} \mid \eta_{\leqq x}\right)=\int v\left(d \bar{\eta}_{>x}\right) \Gamma_{\Lambda}\left(\eta_{<x}, \eta_{x}, \bar{\eta}_{>x}\right) .
$$

By this representation, the difference in (6.9) can be expressed as an integral of $\partial \Gamma_{\Lambda} / \partial \eta_{x}$. Let

$$
W_{x}=\varepsilon \beta \int v\left(d \bar{\eta}_{>x}\right) v\left(d \bar{\eta}_{x}\right) \int_{\bar{\eta}_{x}}^{\eta_{x}} d s \tau_{x}\left(\eta_{<x^{\prime}} s, \bar{\eta}_{>x}\right) .
$$

Formula (6.1) yields

$$
Y_{\Lambda, k}(\eta)=E\left(W_{x_{k}} \mid \mathscr{F}_{\Lambda}\right)
$$

which is particularly convenient since $W_{x}(\eta)$ is independent of $\Lambda$-as long as that region includes $x$.

Since $\left|\tau_{x}\right| \leqq 1$ (by (6.3)), $W_{x}$ obeys:

$$
\left|W_{x}(\eta)\right| \leqq \varepsilon \beta \int v(d \bar{\eta})\left|\eta_{x}-\bar{\eta}\right| \leqq \varepsilon \beta\left[\left|\eta_{x}\right|^{2}+\operatorname{Av}\left(|\eta|^{2}\right)\right]^{1 / 2},
$$

and the similar bound claimed in (6.10).

ii) Next, we prove that (6.11) is satisfied with $b$ defined by

$$
b^{2}=E\left(W_{0}^{2}\right) \text {. }
$$

The proof is based on the ergodic theorem, and some approximation arguments. To get there, we decompose the summand in (6.11), as

$$
\begin{aligned}
E\left(Y_{\Lambda, k}^{2} \mid \mathscr{F}_{\Lambda, k-1}\right)= & E\left(W_{x_{k}}^{2} \mid \eta_{<x_{k}}\right)+\left[E\left(W_{x_{k}}^{2} \mid \mathscr{F}_{\Lambda, k-1}\right)-E\left(W_{x_{k}}^{2} \mid \eta_{<x_{k}}\right)\right] \\
& +E\left(Y_{\Lambda, k}^{2}-W_{x_{k}}^{2} \mid \mathscr{F}_{\Lambda, k-1}\right) .
\end{aligned}
$$

By (6.13) the functions $W_{x}(\eta)$ share the basic covariance properties of $\tau_{x}(\eta)$, i.e.

$$
W_{x}(\eta)=W_{0}\left(T_{-x} \eta\right)
$$

with $T_{x}$ the lattice translation operators. Hence

$$
E\left(W_{x_{k}}^{2} \mid \eta_{<x_{k}}\right)=f\left(T_{-x_{k}} \eta\right)
$$

with $f(\eta) \equiv E\left(W_{0}^{2} \mid \eta<0\right)$. For this term, the ergodic theorem (in its elementary $L^{2}(d v)$ version [28]) implies

$$
\left|\frac{1}{|\Lambda|} \sum_{x \in \Lambda} f\left(T_{x} \eta\right)-b^{2}\right| \underset{L \rightarrow \infty}{\stackrel{d}{\longrightarrow}} 0 .
$$

To bound the effect of the corrections, we let $R$ denote the distance $R(k)=$ $\operatorname{dist}\left(x_{k}, \partial \Lambda\right)$. Simple analysis of the projection operators involved in the conditional 
expectations shows that the other terms in (6.17) can be bounded as follows:

$$
\begin{aligned}
& \left\|E\left(W_{x_{k}}^{2} \mid \eta_{<x_{k}}\right)-E\left(W_{x_{k}}^{2} \mid \mathscr{F}_{\Lambda, k-1}\right)\right\|_{2} \leqq\left\|f-E\left(f \mid \mathscr{F}_{[-R, R]^{d}}\right)\right\|_{2} \equiv a_{1}(R) ; \\
& \left\|E\left(Y_{\Lambda, k}^{2}-W_{x_{k}}^{2} \mid \mathscr{F}_{\Lambda, k-1}\right)\right\|_{1} \leqq 2\left\|W_{0}\right\|_{2}\left\|W_{0}-E\left(W_{0} \mid \mathscr{F}_{[-R, R]}\right)\right\|_{2} \equiv a_{2}(R)
\end{aligned}
$$

with $a_{1}(R), a_{2}(R)$ (defined above) vanishing for $R \rightarrow \infty$. Since the predominant values of $R$, with $k$ averaged over $\{1, \ldots,|\Lambda|\}$, diverge when $L \rightarrow \infty$, the above bounds combined with the triangle inequality(ies) reduce (6.11) to the proven (6.19).

Finally - the bounds (6.6): 1. the upper bound is a consequence of (6.15); (2) for the lower bound [which is more important] we note that by the product structure of $v$, upon differentiation (6.13) yields

$$
\frac{\partial E\left(W_{0} \mid \eta_{0}\right)}{\partial \eta_{0}}=E\left(\tau_{0} \mid \eta_{0}\right)
$$

which —combined with (6.2) and (6.3)—easily implies:

$$
E\left(\frac{\partial E\left(W_{0} \mid \eta_{0}\right)}{\partial \eta_{0}}\right)=M,\left|\frac{\partial E\left(W_{0} \mid \eta_{0}\right)}{\partial \eta_{0}}\right| \leqq 1,\left|\frac{\partial^{2} E\left(W_{0} \mid \eta_{0}\right)}{\partial \eta_{0}^{2}}\right| \leqq \varepsilon \beta \equiv \varepsilon / T .
$$

By the $L^{2}$-contraction property of the conditional expectation, and the definition of the functions $\theta_{v}$ and $\gamma_{v}$ (see (A.3.2)) we now get

$$
b^{2} \equiv E\left(W_{0}^{2}\right) \geqq E\left(\left[E\left(W_{0} \mid \eta_{0}\right)\right]^{2}\right) \geqq \varepsilon^{2} \theta_{v}(M, T / \varepsilon)^{2},
$$

with $\gamma_{v}$ allowed to replace $\theta_{v}$ in case $\tau_{0}(\eta)$ is monotone in $\eta_{0}$.

Proof of Proposition 6.1. Lemma 6.2 establishes the key condition for the applicability of the general theorems presented in Appendix II. Specifically, the moment generating function bound (6.7) and the central limit property (6.5) are implied, respectively, by Propositions A.2.1 (derived there) and A.2.2. These criteria are formulated in the context of double stochastic arrays, which is very suitable for our purpose-as seen by identifying our rectangular regions $\Lambda$ with the index $n$. To further adjust the notation, let

$$
S_{\Lambda, k}(\eta)=\frac{1}{\sqrt{|\Lambda|}} E\left(\Gamma_{\Lambda} \mid \mathscr{F}_{\Lambda, k}\right), \quad \text { and } \quad X_{\Lambda, k}(\eta)=\frac{1}{\sqrt{|\Lambda|}} Y_{\Lambda, k}(\eta) \text {. }
$$

Condition (A.2.3) and the stronger (A.2.14) are established by (6.11). It remains only to verify the condition (A.2.4), for which it suffices to verify the unconditional form of the Lindeberg property stated there. To do that, we first invoke the Jensen inequality - applicable here by the convexity of the function $\Phi(X)=X^{2} I[X>a]$, and the relation (6.14), by which

$$
E\left(X_{\Lambda, i}^{2} I\left[\left|X_{\Lambda, i}\right|>a\right]\right) \leqq E\left(\frac{1}{|\Lambda|} W_{0}^{2} I\left[\left|W_{0}\right|>a \sqrt{|\Lambda|}\right]\right) .
$$

Hence we have:

$$
E\left(U_{\Lambda, a}\right) \sum_{i=1}^{|A|} E\left(X_{\Lambda, i}^{2} I\left[\left|X_{\Lambda, i}\right|>a\right]\right) \leqq E\left(W_{0}^{2} I\left[W_{0}>a \sqrt{|\Lambda|}\right]\right) \rightarrow 0 \text { for }|\Lambda| \rightarrow \infty,
$$


with the last statement implied by the square integrability of $\left|W_{0}\right|$ (guaranteed by (6.15)).

\section{The Rounding Effect Systems with Continuous Symmetries in $d \leqq 4$ Dimensions}

In this section we focus on systems which are stochastically invariant under the action of a continuous, connected group -in the sense explained in Sect. 4c.

With the goal of proving Theorem 4.4, we assume the system has all the features listed in Sect. $4 \mathrm{c}(\mathrm{i}-\mathrm{v})$. The symmetry group, $\mathscr{G}$, is a connected, closed (with no loss of generality there), subgroup of $O(N)$. The key step is to prove the following lemma, which is in fact an equivalent formulation of Theorem 4.4-cast in a statistical mechanical terminology.

Lemma 7.1. In a system having the features i)-v) described in Sect. $4 c$, let $\rho_{\eta}$ be a function associating to each $\eta$ (in a set of full measure) a Gibbs state for the corresponding interaction, with $\underline{h}=\underline{0}$, which is the baricenter of a measure $\mu_{\eta}(\cdot)$ having the properties i)-iv) listed in Proposition 5.1. If $d \leqq 4$ ( $d$ being the dimension of the lattice), then the mean magnetization $(\underline{m})$ is $\mathscr{G}$-invariant:

$$
\underline{m}=\int v(d \eta) \rho_{\eta}\left(\underline{\sigma}_{0}\right) \in I_{\mathscr{G}},
$$

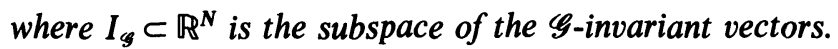

Remark. The conclusion of Theorem 4.4 implies (7.1) for any family of $\eta$-dependent Gibbs states which are weak* measurable, and translation-covariant (in the sense of (5.3)).

Our proof utilizes the framework developed in this paper, incorporating ideas which have been developed in the analysis of the classical Mermin-Wagner phenomenon [20]. In the background is an argument of Herring-Kittel [21], which was elucidated by Pfister in his rigorous proof of that two-dimensional phenomenon [22]. We make use also of related observations by Dobrushin and Shlosman [23], Fannes, Vanheuverzwijn and Verbeure [24], and Georgii [25].

The meaning of $(7.1)$ is that

$$
\underline{m}=R \underline{m}
$$

for each $R \in \mathscr{G}$. It suffices to prove (7.2) under the assumption that $R$ is a cyclic element, meaning here that it belongs to a closed one-parameter subgroup of $\mathscr{G}$, isomorphic to the circle. The reason is that the union of such subgroups is dense in $\mathscr{G}$, or better yet: each element of $\mathscr{G}$ can be written as a product of cyclic elements (with $\leqq N(N-1) / 2$ factors). [Each $R \in \mathscr{G}$ belongs to a one-parameter subgroup. If the subgroup is closed it is isomorphic to the circle. If not, then its closure is isomorphic to a finite dimensional torus, [26].]

To prove Lemma 7.1 we now fix the attention on a specific measure $\mu_{\eta}(\cdot)$ [a function of $\eta$ with values in the space of probability measures on the space of states - with the baricenter $\left.\rho_{\eta}\right]$ having the properties listed in Proposition 5.1. We specify also the symmetry map $R$, assumed to belong to a closed one-parameter subgroup of $\mathscr{G}$. 
For the specified $\mu_{\eta}$ and $R$, let us define the measures $\hat{\mu}_{\eta}(d \rho)$ by:

$$
\int \hat{\mu}_{\eta}(d \rho) \rho(f(\sigma))=\int \mu_{R^{-1} \eta}(d \rho) \rho(f(R \sigma)), \quad \text { for all "observables" } f,
$$

where $R^{-1} \eta$ and $R \sigma$ are the uniformly rotated configurations. Upon a moment of reflection, one can easily see that $\hat{\mu}_{\eta}(\cdot)$ is supported within the collection of Gibbs states for the interaction $H_{\eta, R}$, with the specified random fields $\eta$ and the uniform field rotated to $R \underline{h}$. In its dependence on $\eta$, the new measure inherits the covariance properties of Proposition 5.1. If $\underline{h}=\underline{0}$, as is the case considered here, then $\hat{\mu}_{\eta}(\cdot)$ and $\mu_{\eta}(\cdot)$ are supported in the set of Gibbs states for the same interaction, though possibly with different boundary conditions.

For these two measures we have:

and

$$
\int v(d \eta) \int \mu_{\eta}(d \rho) \rho\left(\underline{\sigma}_{0}\right)=\underline{m},
$$

$$
\int v(d \eta) \int \hat{A}_{\eta}(d \rho) \rho\left(\underline{\sigma}_{0}\right)=R \underline{m},
$$

where (7.4a) is an explicit statement of (7.1), and (7.4b) is by (7.3) and the $\mathscr{G}$-invariance of $v(d \eta)$. Following the general strategy of this paper, in order to prove (7.2) we focus on the fluctuations of the auxiliary quantity $G_{A}(\eta)$, which for this section is defined as:

$$
G_{\Lambda}(\eta)=\frac{1}{2 \beta} \int \hat{\mu}_{\eta}(d \rho) \ln \rho\left(e^{-\beta \varepsilon \eta_{\Lambda} \cdot \sigma_{\Lambda}}\right)-\frac{1}{2 \beta} \int \mu_{\eta}(d \rho) \ln \rho\left(e^{-\beta \varepsilon \eta_{\Lambda} \sigma_{\Lambda}}\right),
$$

where $\underline{\eta}_{A} \cdot \underline{\sigma}_{A} \equiv \sum_{n \in A} \underline{\eta}_{x} \cdot \underline{\sigma}_{x}$. As in (5.16), we define $\tilde{G}_{\Lambda}\left(\eta_{A}\right)=E\left(G_{A} \mid \eta_{A}\right)-E\left(G_{A}\right)$.

By now, the reader will not be surprised to learn that $\tilde{G}_{\Lambda}\left(\eta_{\Lambda}\right) / \sqrt{|\Lambda|}$ has in the limit a normal distribution (a fact implied by Proposition 6.1), with variance which does not vanish if (7.2) is violated (see below). The new information, which raises the marginal dimension to $d=4$, is that under the assumptions made in Theorem 4.4, $\tilde{G}_{\Lambda}\left(\eta_{\Lambda}\right)$ obeys the uniform bound (for $\left.\Lambda=[-L, L]^{d}\right)$ :

$$
\left|\tilde{G}_{\Lambda}\left(\eta_{\Lambda}\right)\right| \leqq \text { const. } L^{d-2}+B \sqrt{|\Lambda|},
$$

where $B=0$ for finite range interactions. The exponent in the first term is an improvement over $L^{d-1}$ seen in the general (1.3). Following is the key step in the derivation of (7.6).

Proposition 7.2. For the given $R \in \mathscr{G}$, assume there is some $s \in[0,1]$ and a pair of transformations on the space of configurations $\tilde{R}_{i}, i=1,2$, which act as products of single-site rotations by elements of $\mathscr{G}\left(\widetilde{R}_{i}=\otimes R_{\theta_{x}, i}\right)$, such that:

1) for both $i=1$ and $2, \widetilde{R}_{i}$ acts as $R$ in $\Lambda$ and as the identity map in the complement of some finite region $V(\supseteqq \Lambda)$,

2) there is some $K_{A}(R)$, with which (uniformly in $\sigma$ )

$$
s\left[H_{0}\left(\tilde{R}_{1} \sigma\right)-H_{0}(\sigma)\right]+(1-s)\left[H_{0}\left(\tilde{R}_{2} \sigma\right)-H_{0}(\sigma)\right] \leqq K_{A}(R) .
$$

Then $\tilde{G}_{\Lambda}$, defined above, obeys the uniform bound

$$
\tilde{G}_{\Lambda}\left(\eta_{\Lambda}\right) \leqq K_{\Lambda}(R) .
$$

In the following discussion, we refer by the term local rotation to a map acting 
as a product of rotations-at-a-site, by elements of $\mathscr{G}$ which differ from the identity at only a finite number of sites. We reserve the symbol $\tilde{R}$ for such a map.

To prove the proposition we first establish a pair of lemmas.

Lemma 7.3. Let $\rho_{\eta}(d \sigma)$ be a Gibbs state for the Hamiltonian $H_{\eta}(\sigma)=H_{0}(\sigma)-\varepsilon \eta \cdot \sigma$, where $\eta \cdot \sigma \equiv \sum_{x} \eta_{x} \cdot \underline{\sigma}_{x} ;$ with $H_{0}(\sigma)$ and the a-priori measure $\rho_{0}(d \sigma)$ both $\mathscr{G}$-invariant. If $\tilde{R}$ is a local rotation, then for every local spin function $g$ :

$$
\rho_{\eta}\left(e^{\beta(\tilde{R} \eta-\eta) \cdot \sigma} g(\sigma)\right)=\rho_{\eta}\left(e^{\beta\left[H_{0}(\sigma)-H_{0}(\tilde{R} \sigma)\right]} g(\tilde{R} \sigma)\right)
$$

(with the terms in the exponents interpreted as sums of only the nonzero terms-of which there is only a finite number).

Proof. Let $\rho_{0}$ be the Gibbs state obtained from $\rho_{\eta}$ by the removal from the Gibbs factor (in the natural sense) all the Hamiltonian terms involving spins in $V$-which is a finite region outside of which $\tilde{R}$ acts as the identity. That state is invariant under $\tilde{R}$, and thus (in a hopefully clearly understood notation):

$$
\begin{aligned}
\rho_{\eta}\left(e^{\beta(\tilde{R} \eta-\eta) \cdot \sigma} g(\sigma)\right) & =\rho_{0}\left(e^{-\beta\left[H_{0}(\sigma)-\tilde{R} \eta \cdot \sigma\right]_{V}} g(\sigma)\right) / Z_{V}(\eta) \\
& =\rho_{0}\left(e^{-\beta\left[H_{0}(\tilde{R} \sigma)-\tilde{R} \eta \cdot \tilde{R} \sigma\right]_{V}} g(\tilde{R} \sigma)\right) / Z_{V}(\eta) \\
& =\rho_{0}\left(e^{-\beta\left[H_{0}(\tilde{R} \sigma)-\eta \cdot \sigma\right]_{V}} g(\tilde{R} \sigma)\right) / Z_{V}(\eta) \\
& =\rho_{\eta}\left(e^{\beta\left[H_{0}(\sigma)-H_{0}(\tilde{R} \sigma)\right]} g(\tilde{R} \sigma)\right)
\end{aligned}
$$

with $Z_{V}(\eta)=\rho_{0}\left(e^{-\beta\left[H_{0}(\sigma)-\eta \cdot \sigma\right]_{V}}\right)$.

Lemma 7.4. If $H_{0}(\sigma)$ and the a-priori measure $\rho_{0}(d \sigma)$ are both $\mathscr{G}$-invariant, then for each $R \in \mathscr{G}$ and $\mu_{\eta}(d \rho)$ as above:

i)

$$
E\left(G_{A}\right) \equiv \int v\left(d \eta_{\Lambda}\right) G_{\Lambda}(\eta)=0 .
$$

and $\tilde{G}_{A}\left(=E\left(G_{A} \mid \eta_{A}\right)-E\left(G_{A}\right)\right)$ admits the representation

ii)

$$
\tilde{G}_{\Lambda}\left(\eta_{\Lambda}\right)=\frac{-1}{2 \beta} \int v\left(d \eta_{\Lambda}\right) \int \mu_{\eta}(d \rho) \ln \rho\left(e^{\beta\left[H_{0}(\sigma)-H_{0}\left(\tilde{R}^{-1} \sigma\right)\right]}\right)
$$

and satisfies the bound:

$$
\tilde{G}_{\Lambda}\left(\eta_{A}\right) \leqq \frac{1}{2} \int v\left(d \eta_{A^{c}}\right) \int \mu_{\eta}(d \rho) \rho\left(H_{0}\left(\tilde{R}^{-1} \sigma\right)-H_{0}(\sigma)\right),
$$

with $\tilde{R}$ any local rotation which acts as $R$ throughout $\Lambda$ (and as the identity in the complement of some finite $V$ ).

Proof. Combining the definitions of $G_{A}$ and $\hat{\mu}_{\eta},(7.5)$ and (7.3), we get

$$
G_{\Lambda}(\eta)=\frac{1}{2 \beta} \int \mu_{R^{-1} \eta}(d \rho) \ln \rho\left(e^{-\beta \varepsilon R^{-1} \underline{\eta}_{\Lambda} \cdot \underline{\sigma}_{\Lambda}}\right)-\frac{1}{2 \beta} \int \mu_{\eta}(d \rho) \ln \rho\left(e^{-\beta \varepsilon \underline{\eta}_{\Lambda} \cdot \underline{\sigma}_{\Lambda}}\right) .
$$

By the $\mathscr{G}$-invariance of $v(d \eta),(7.14)$ implies (7.11) and also the following representation

$$
E\left(G_{\Lambda} \mid \eta_{\Lambda}\right)=\frac{1}{2 \beta} \int v\left(d \eta_{\Lambda} c\right)\left[\int \mu_{\tilde{R}^{-1} \eta^{\prime}}(\mathrm{d} \rho) \ln \rho\left(e^{-\beta \varepsilon R^{-1} \eta_{\Lambda} \cdot \sigma_{\Lambda}}\right)-\int \mu_{\eta}(d \rho) \ln \rho\left(e^{-\beta \varepsilon \eta_{\Lambda} \cdot \underline{\sigma}_{\Lambda}}\right)\right]
$$

where $\underline{\eta}_{\Lambda} \cdot \underline{\sigma}_{\Lambda} \equiv \sum_{x \in \Lambda} \underline{\eta}_{x} \cdot \underline{\sigma}_{x}$ 
Using the transformation law (5.7) - which describes the local dependence of $\mu_{\eta}$ on $\eta$, we may reduce the above expressions to $\mu_{\eta_{v e}}(d \rho)$-where $V$ is the region outside of which $\widetilde{R}^{-1}$ acts as the identity. ( $\eta_{V c}$ is the configuration obtained from $\eta$ by setting it to zero in $V$.) We get

$$
2 \beta \tilde{G}_{\Lambda}\left(\eta_{\Lambda}\right)=\int v\left(d \eta_{\Lambda^{c}}\right) \int \mu_{\eta_{V c}}(d \rho)\left[\ln \frac{\rho\left(e^{\beta \varepsilon \tilde{R}^{-1} \underline{\eta}_{V / \Lambda} \tilde{\sigma}_{V / \Lambda}}\right)}{\rho\left(e^{\beta \varepsilon \tilde{R}^{-1} \eta_{V^{\prime}} \underline{\sigma}_{V}}\right)}-\ln \frac{\rho\left(e^{\beta \varepsilon \eta_{V / \Lambda} \cdot \underline{\sigma}_{V / \Lambda}}\right)}{\rho\left(e^{\beta \varepsilon \eta_{V^{\prime}} \underline{\sigma}_{V}}\right)}\right] .
$$

The exponents in the two numerators do not involve the specified $\eta_{A}$. By the $\mathscr{G}$-invariance of $v\left(d \eta_{A^{c}}\right)$, the integrals of the two corresponding terms cancel. The terms in the denominators may be combined by a reversed application of (5.7). That yields:

$$
2 \beta \tilde{G}_{\Lambda}\left(\eta_{\Lambda}\right)=-\int v\left(d \eta_{A^{c}}\right) \int \mu_{\eta}(d \rho) \ln \rho\left(e^{\left.\beta \varepsilon \tilde{R}^{-1} \eta_{V}-\eta_{V}\right) \cdot \sigma_{V}}\right) .
$$

The claimed (7.12) follows now by an application of Lemma 7.3, for which we note that $\left(\tilde{R}^{-1} \underline{\eta}_{V}-\underline{\eta}_{V}\right) \cdot \sigma_{V}=\left(\tilde{R}^{-1} \underline{\eta}-\underline{\eta}\right) \cdot \underline{\sigma}\left(\right.$ since $\tilde{R}^{-1}$ acts as the identity in $\left.V^{c}\right)$.

The bound (7.13) is a simple consequence of (7.12) and the Jensen inequality, $\rho\left(e^{X}\right) \geqq \exp (\rho(X))$.

Proof of Proposition 7.2. Let $\widetilde{R}_{i}, i=1$ and 2, be a pair of local rotations satisfying the stated assumptions. Through (7.13), each of them yields a bound for $\widetilde{G}_{A}\left(\eta_{\Lambda}\right)$. Taking a weighted mean of the two, we get

$$
\begin{aligned}
\tilde{G}_{\Lambda}\left(\eta_{\Lambda}\right) & \leqq \frac{1}{2} \int v\left(d \eta_{A^{c}}\right) \int \mu_{\eta}(d \rho) \rho\left(s\left[H_{0}\left(\tilde{R}_{1}^{-1} \sigma\right)-H_{0}(\sigma)\right]+(1-s)\left[H_{0}\left(\tilde{R}_{2}^{-1} \sigma\right)-H_{0}(\sigma)\right]\right) \\
& \leqq K_{\Lambda} / 2,
\end{aligned}
$$

where we invoked the hypothesis (7.7).

Lemma 7.5. Let $H$ be an interaction (4.4) satisfying the smoothness condition (4.5), and let $R$ be an element of a closed one parameter subgroup of $\mathscr{G}$, of period $P$ (in the natural parametrization described above (4.5)). Then, for every $\Lambda=[-L, L]^{d}$ there exists a pair of local rotations, $\widetilde{R}_{i}, i=1,2$, which act as $R$ in $\Lambda$ and as the identity in the complement of the finite set $V=[-2 L, 2 L]^{d}$, with which the following bound holds for all spin configurations $\sigma$ :

$$
s\left[H_{0}\left(\tilde{R}_{1}^{-1} \sigma\right)-H_{0}(\sigma)\right]+(1-s)\left[H_{0}\left(\tilde{R}_{2}^{-1} \sigma\right)-H_{0}(\sigma)\right] \leqq 4^{d} P^{2}\left(\sum_{z \in \mathbf{Z}^{d}} J_{z}|z|^{2}\right) L^{d-2},
$$

where $s=s(R) \in[0,1]$. If the sum on the right side diverges, but $d<4$ and the interaction satisfies (4.6) then a modified statement holds — with the right side in (7.19) replaced by $P^{2}$ Const. $L^{d / 2}$.

Proof. Let us denote the elements of the one-parameter subgroup as $\left\{\boldsymbol{R}_{\theta}\right\}$ with $\theta \in \mathbb{R}_{\text {mod. } P}$-in the parametrization of (4.5). In particular: $R=R_{s p}$, for some $s \in[0,1]$. For a given $L$, we choose $\tilde{R}_{i}, i=1,2$ to be the local rotations:

$$
\left(\tilde{R}_{i} \sigma\right)_{x}=R_{\theta_{x, i}} \sigma_{x}
$$


with the angles $\theta_{x, i}$ defined by:

$$
\begin{aligned}
& \theta_{x, 1}=-(1-s) P \cdot W_{L, x} \\
& W_{L, x} \text { and } \begin{array}{cc}
\theta_{x, 2}=s P \cdot W_{L, x}, \\
\frac{(1+r) L-\|x\|}{r L} & L<\| \leqq L \\
0 & (1+r) L<\|x\|
\end{array}
\end{aligned}
$$

$\|x\|=\sum_{k=1}^{d}\left|x_{k}\right|$. For concreteness sake we take $r=1$.

The energy differences $H_{0}\left(\tilde{R}_{i}^{-1} \sigma\right)-H_{0}(\sigma)$ with $i=1,2$, are sums of

$$
\begin{aligned}
\Delta_{x, y} \Psi & \equiv \Psi_{x-y}\left(R_{-\theta_{x, i}} \sigma_{x}, R_{-\theta_{y, i}} \sigma_{y}\right)-\Psi_{x-y}\left(\sigma_{x}, \sigma_{y}\right) \\
& =\Psi_{x-y}\left(R_{\left(\theta_{y, i}-\theta_{x, i}\right.} \sigma_{x}, \sigma_{y}\right)-\Psi_{x-y}\left(\sigma_{x}, \sigma_{y}\right),
\end{aligned}
$$

where we applied the rotation-invariance of $\Psi$. Using Taylor's expansion to the second order, and the bound (4.5) on the second derivative, we have:

$$
\left|\Delta_{x, y} \Psi-\left(\theta_{y, i}-\theta_{x, i}\right) A \Psi_{x-y}\left(\sigma_{x}, \sigma_{y}\right)\right| \leqq \frac{1}{2}\left(\theta_{y, i}-\theta_{x, i}\right)^{2},
$$

where $A$ is a certain linear operator (related to the generator of the one-parameter subgroup). The contributions of the first-order terms to the left side of (7.19) cancel, since

$$
s\left(\theta_{y, 1}-\theta_{x, 1}\right)+(1-s)\left(\theta_{y, 2}-\theta_{x, 2}\right) \equiv 0
$$

Hence,

$$
\begin{aligned}
& s\left[H_{0}\left(\tilde{R}_{1}^{-1} \sigma\right)-H_{0}(\sigma)\right]+(1-s)\left[H_{0}\left(\tilde{R}_{2}^{-1} \sigma\right)-H_{0}(\sigma)\right] \\
& \leqq \sum_{\substack{x y: \\
L \leqq\|x\| \leqq 2 L}}\left|J_{x-y}\right| P^{2} \max \left\{\frac{\|x-y\|^{2}}{(2 L)^{2}}, 1\right\},
\end{aligned}
$$

from which the claimed (7.18) readily follows.

Putting the above results together, we reach the main goal of this section.

Proof of Lemma 7.1. As explained above, it suffices to prove (7.2) for cyclic rotations $R \in \mathscr{G}$. For such $R$, Proposition 7.2 and Lemma 7.5 imply the uniform bound

$$
\left|\tilde{G}_{\Lambda}\left(\eta_{\Lambda}\right)\right| \leqq \text { Const. } L^{d-2}+B \sqrt{|\Lambda|}
$$

with the second term absent for short range interactions. Concurrently, the quantity $\widetilde{G}_{A}\left(\eta_{A}\right)$ satisfies the assumptions of the multicomponent version of Proposition 6.1 - which requires only the obvious adjustments. For the present case:

$$
\operatorname{Av}\left(\frac{\partial \tilde{G}_{\Lambda}\left(\eta_{\Lambda}\right)}{\partial \eta_{x}^{j}}\right)=\varepsilon M_{j}, \quad \text { with } \quad \underline{M}=R \underline{m}-\underline{m} .
$$

Hence, $\tilde{G}_{\Lambda}\left(\eta_{\Lambda}\right)$ has a Gaussian limit:

$$
\tilde{G}_{\Lambda}\left(\eta_{\Lambda}\right) / \sqrt{|\Lambda|} \underset{L \rightarrow \infty}{\stackrel{D}{\longrightarrow}} N\left(0, b^{2}\right)
$$


with

$$
b \geqq \varepsilon \theta_{v}^{(N)}(\underline{M}, T / \varepsilon),
$$

where $\theta_{v}^{(N)}(\underline{M}, T / \varepsilon)$ is the function discussed in Appendix IIIb. As is shown there, under the continuity assumptions made on $v, \theta_{v}^{(N)}(\underline{M}, T / \varepsilon)$ vanishes only at $\underline{M}=0$. Hence, a contradiction is avoided only if $\underline{M}=\underline{0}$, i.e. if (7.2) is satisfied.

Proof of Theorem 4.4. If at $h=0$ the system's free energy, $F(T, \underline{h}, \varepsilon)$, has a nonvanishing directional derivative in some direction $v \in \mathbb{R}^{N}$, then there is a function associating to each configuration of the random couplings $\eta$ a Gibbs state, $\rho_{\eta}$, such that:

$$
\int v(d \eta) \rho_{\eta}\left(\underline{v} \cdot \underline{\sigma}_{0}\right)=\beta D(\underline{v}) F(T, \underline{h}, \varepsilon) \neq 0 .
$$

Furthermore, by the construction of Appendix I, one may realize (7.30) with the state $\rho_{\eta}$ being a baricenter of a probability measure $\mu_{\eta}$ (on the space of states) with the properties listed in Proposition 5.1. Now, if the direction vector $\underline{v}$ is not orthogonal to the collection of $\mathscr{G}$-invariant vectors, then (assuming $d \leqq 4$, and the other features listed in Theorem 4.4), Eq. (7.30) is in contradiction with Lemma 7.1. Hence, the directional derivatives vanish in all the directions in the orthogonal complement of $I_{\mathscr{g}}$, as stated in (4.9).

\section{Appendix I. Gibbs States for Random Systems}

In this appendix we construct the $\eta$-dependent measure $\mu_{\eta}$ on the space of states, which is instrumental for the general version of the main result. We are not aware of a general construction of a map associating to each (typical) random coupling configuration a single Gibbs state $\rho_{\eta}$ with all the covariance properties listed in Proposition 5.1. In fact, there are grounds to question the general existence of such a map.

a. Topological Preliminaries. We denote by $(\Omega, \mathscr{B})$ the measurable space of spin configurations. As in Sect. 3, we restrict here the discussion to systems whose single-spin space may be endowed with a topology (such that $\mathscr{B}$ is the corresponding Borel $\sigma$-field) in which the interactions are continuous, and the space is Hausdorff, compact and separable - in the sense that it has a countable base. [Such a topological space is metrizable, and admits a countable collection of continuous functions which generate the topology.] Further generalizations are possible, e.g. within the setup discussed in [25] - but they require some other assumptions.

Let $\mathscr{P}(\Omega, \mathscr{B}) \equiv \mathscr{P}$ be the space of Borel probability measures on $\Omega$ endowed with the $\mathscr{W}$-topology induced by the convergence of integrals of bounded continuous functions (to which probability texts refer to as the weak topology [27], and functional analysis texts as the weak* topology [28]). The elements of $\mathscr{P}$ are the states of the spin system. For reasons indicated above, we shall associated to each (typical) random coupling configuration $\eta$ not a single state, but a probability measure on the space of states (whose baricenter, (5.10), is however an element of $\mathscr{P})$. We consider therefore also the space $\mathscr{M}$ of probability measures 
on $\mathscr{P}$, endowed with the $\mathscr{W}$-topology induced by the convergence of integrals of (bounded) continuous functions of the states.

The basic relevant fact is that the space of probability measures on a compact separable space is (in its $\mathscr{W}$-topology) also compact and separable [27]. (The compactness can be viewed as the separable case of the more general BanachAlaoglu theorem [28].) Thus, the topologies we specified for the spaces $\mathscr{P}$ and $\mathscr{M}$ inherit the properties we listed for $\boldsymbol{\Omega}$.

For more concrete convergence criteria, one may choose a countable collection of functions $f_{n}$ on $\Omega$, and a countable collection of functions $g_{m, N}: \mathbb{R}^{N} \rightarrow \mathbb{R}$, $N=1,2, \ldots$, such that $\left\{f_{n}\right\}_{n}$ and $\left\{g_{m, N}\right\}_{m}$ are dense in the uniform topologies of $\mathbb{R}$ and, respectively, $\mathbb{R}^{N}$. Applying the Stone-Weierstrass theorem [28], it is easy to see that for each such choice:

i) a sequence $\rho_{k} \in \mathscr{P}$ converges to $\rho \Leftrightarrow$ for each $n, \rho_{k}\left(f_{n}\right) \underset{k}{\longrightarrow} \rho\left(f_{n}\right)$,

ii) a sequence $\mu_{k} \in \mathscr{M}$ converges to $\mu \Leftrightarrow$ for each $k, N<\infty$, and each finite collection $f_{n_{1}}, \ldots, f_{n_{N}}$,

$$
\int g_{m, N}\left(\rho\left(f_{n_{1}}\right), \ldots, \rho\left(f_{n_{N}}\right)\right) d \mu_{k} \underset{k}{\longrightarrow} \int g_{m, N}\left(\rho\left(f_{n_{1}}\right), \ldots, \rho\left(f_{n_{N}}\right)\right) d \mu
$$

These topologies are metrizable-e.g. with the metrics which are easy to generate from the above criteria.

We now turn to the topology of the interactions, and the space of their functions. In setting it, we are attempting to capture two different notions. On one hand, various statements concerning random interactions are true only for "typical" $\eta$, and they are expressed as holding only $v$-almost surely. At the same time, in order to differentiate in $\eta_{x}$ (or express the very important property of $\eta$-covariance) the claimed properties should be stable under arbitrary local changes of $\eta$-even if the support of $v\left(d \eta_{\alpha, x}\right)$ is a strict subset of $\mathbb{R}$. The two notions may be merged by narrowing the clause of typicality to $\eta$ 's behaviour at infinity.

Let $\mathscr{H}$ denote the set of configurations of the random couplings $\eta=\left\{\eta_{\alpha, x}\right\}$. There are two relevant topologies on $\mathscr{H}$.

- The product topology $\mathscr{T}_{p}$, whose Borel sets define the $\sigma$-algebra on which the probability $v(d \eta)$ is defined, and

-The topology $\mathscr{T}_{N}$ generated by the metric associated with:

$$
\left\|\eta^{\prime}-\eta\right\| \equiv \sum_{\alpha, x} \varepsilon_{\alpha}\left|\eta_{\alpha, x}^{\prime}-\eta_{\alpha, x}\right| \text {. }
$$

Functions which are continuous in $\mathscr{T}_{N}$ need not be continuous in the product topology $\mathscr{T}_{\boldsymbol{P}}$. The latter is also metrizable, though not by a translation invariant metric. The price of translation invariance in (A.1.2) is $\mathscr{T}_{N}$ 's lack of separability.

We denote by $\mathscr{L}(\mathscr{H}, \mathbb{R})$ the space of real-valued functions on $\mathscr{H}$ having the two properties:

-measurability (in the Lebesgue sense) with respect to the $\sigma$-algebra associated with $\mathscr{T}_{\boldsymbol{P}}$

- continuity under local changes in $\eta$.

More precisely, $\Psi \in \mathscr{L}(\mathscr{H}, \mathbb{R})$ if for every finite region $\Lambda, \Psi$ has a version of the form $\Psi\left(\eta_{\Lambda}, \eta_{\Lambda^{c}}\right)$ which is continuous in $\left.\eta_{\Lambda} \equiv\left\{\eta_{\alpha, x} \mid T_{x} \alpha \subset \Lambda\right\}\right)$. With this choice, we 
denote

$$
E\left(\Psi \mid \eta_{A}\right)=\int v\left(d \eta_{A^{c}}^{\prime}\right) \Psi_{k}\left(\eta_{A}, \eta_{A^{c}}^{\prime}\right)
$$

Our choice of the topology on $\mathscr{L}(\mathscr{H}, \mathbb{R})$ is the one for which the convergence of functions, $\Psi_{k} \rightarrow \hat{\Psi}$ means that

$$
E\left(\Psi \mid \eta_{A}\right) \underset{k \rightarrow \infty}{\longrightarrow} E\left(\hat{\Psi} \mid \eta_{A}\right)
$$

for every finite lattice subset $\Lambda$, and for all $\eta$.

With the analogous conventions, we define the topological space $\mathscr{L}(\mathscr{H}, \mathscr{M})$, of $\mathscr{M}$-valued functions of $\eta$.

Lemma A.1.1. Let $Q \subset \mathscr{L}(\mathscr{H}, \mathbb{R})$ be a collection of uniformly bounded functions. If for any $\Lambda$, the set of projections $\left\{E\left(\Psi \mid \eta_{\Lambda}\right) \mid \Psi \in Q\right\}$ is compact, then any sequence of elements of $Q$ has a convergent subsequence (with a limit in $\mathscr{L}(\mathscr{H}, \mathbb{R})$ ). A similar statement holds in $\mathscr{L}(\mathscr{H}, \mathscr{M})$.

Proof. Let $\Psi_{k}(\eta)$ be a sequence of functions in $Q$. By the assumed compactness, and the diagonal trick, there is a subsequence for which the functions $E\left(\Psi_{k_{j}} \mid \eta_{A}\right)$ converge-simultaneously for all finite $\Lambda$. We denote the limits as $\hat{\Psi}_{\Lambda}(\eta)$. These functions satisfy a consistency condition which may be read as saying that $\hat{\Psi}_{A}(\eta)$ form a martingale with respect to the sequence of $\sigma$-algebras generated by $\eta_{\Lambda}$. By Doob's martingale convergence theorem [29], there exists a function $\hat{\Psi}(\eta)$ - to which $\hat{\Psi}_{\Lambda}(\eta)$ converge for $v$-a.e. $\eta$, such that $E\left(\hat{\Psi} \mid \eta_{\Lambda}\right)=\hat{\Psi}_{\Lambda}(\eta)=\lim _{j \rightarrow \infty} E\left(\Psi_{k_{j}} \mid \eta_{\Lambda}\right)$. According to our definition of convergence, (A.1.1), that means $\hat{\Psi}=\lim _{j \rightarrow \infty}^{j \rightarrow \infty} \Psi_{k_{j}}$.

For $Q$ a subset of $\mathscr{L}(\mathscr{H}, \mathscr{M})$, the proof is by an argument like the above-with the addition of the Riesz-Markov theorem [28], which identifies the elements of $\mathscr{M}$ with positive linear functionals on $C(\mathscr{P})$.

\section{b. The Construction of $\mu_{\eta}$.}

Proof of Proposition 5.1. The proof consists of a construction, carried out in three steps, and the verification of the desired properties. In the first two steps of the construction we define finite-volume objects with a restricted version of the $\eta$-covariance. The values of $(T, h, \varepsilon)$ are fixed throughout the discussion.

1. Let $\rho_{\eta, K}$ denote the periodic-boundary (p.b.) Gibbs state in $[-K, K]^{d}$, for the indicated $\eta$, crossed with the a priori product measure in the complement of that set. [In defining the p.b. state, we exclude from the interaction the terms $\Phi_{A}$ and $g_{\alpha}$ with $\operatorname{diam}(A), \operatorname{diam}(\alpha) \geqq 2 K$, and periodicize both the homogeneous and the random parts of the interaction.] The extension of the state to the infinite system allows us the convenience of regarding $\rho_{\eta, K}$ as an element of $\mathscr{P}$, which we view as a function of all $\eta$-even though it depends only on $\eta$ 's restriction to $[-K, K]^{d}$. For $\Delta \eta$ supported in $[-K, K]^{d}$ we have

$$
\rho_{\eta+\Delta \eta, K}(-)=\rho_{\eta, K}\left(-e^{-\beta \Delta H}\right) / \rho_{\eta, K}\left(e^{-\beta \Delta H}\right) \equiv U_{\Delta \eta} \rho_{\eta, K},
$$

with $\Delta H(\sigma) \equiv \sum_{\alpha, x} \varepsilon_{\alpha} \Delta \eta_{\alpha, x} g_{\alpha, x}$. It should be noted that for each bounded function on $\Omega$,

$$
\left|U_{\Delta \eta} \rho(f)-\rho(f)\right| \leqq\|\Delta \eta\| \cdot \sup |f(\sigma)| .
$$


In particular, for compactly supported $\Delta \eta$, the map $U_{\Delta \eta}: \mathscr{P} \rightarrow \mathscr{P}$ is continuous. 2. We now consider the collection of "windows" (on $\eta$ ), provided by the rectangular regions $W \subset[-K, K]^{d}$. Keeping the restriction of $\eta$ to a given $W$ fixed $\left(\eta_{W} \equiv\left\{\eta_{\alpha, x} \mid T_{x} \alpha \subset W\right\}\right)$, let us regard $\rho_{\eta, K}$ as a random state, with the probability measure induced for it by $v\left(d \eta_{W^{c}}\right)$. The resulting probability measure on $\mathscr{P}$ is denoted by $\mu_{\eta}^{W ; K}$. As a function of $\eta, \mu_{\eta}^{W ; K}$ depends on it only through $\eta_{W}$. It has the following properties:

i) Consistency: for each $W \subset W^{\prime} \subset[-K, K]^{d}$,

$$
\mu_{\eta}^{W ; K}=\int v\left(d \tilde{\eta}_{W^{c}}\right) \mu_{\left(\eta_{W}, \tilde{\eta}_{W c}\right)}^{W^{\prime} ; K}
$$

ii) Partial translation-covariance: let $T_{x}$ be the translation by $x$, defined consistently in the various spaces considered here. Then

$$
\int \mu_{T_{x} \eta}^{T_{x} W ; K}(d \rho) \Psi(\rho)=\int \mu_{\eta}^{W ; K}(d \rho) \Psi\left(T_{x} \rho\right)
$$

for each continuous function on the state space $\mathscr{P}, \Psi(\rho)$, which depends only on $\rho$ 's restriction to a set $A$ with $A \subset T_{x} W \subset[-K, K]^{d}$.

iii) Response to local changes: for each $\Delta \eta$ supported in $W \subset[-K, K]^{d}$ we have

$$
\mu_{\eta+\Delta \eta}^{W ; K}(d \rho)=\mu_{\eta}^{W ; K}\left(d U_{\Delta \eta}^{-1} \rho\right) .
$$

It follows from (A.1.6) that $\mu_{\eta}^{W ; K}$ are uniformly continuous functions of $\eta_{W}$. [Since the target space, $\mathscr{M}$, is compact, the notion of uniform continuity is independent of the metric chosen for $\mathscr{M}$. Some specific metrics are suggested by the explicit convergence criterion (A.1.1).]

3. We now make the physically relevant choice of the subsequence of volumes for which the infinite-volume limit is constructed.

By the separability of $\mathscr{P}$, and the diagonal sequence trick, it is possible to produce for the given system a subsequence of values $K \rightarrow \infty$ for which $\mu_{\eta}^{W ; K}$ converge -in the $\mathscr{W}$ topology of $\mathscr{M}$, simultaneously for all rectangular $W$ with the initial choice $\eta=0$. Since for each such (finite) $W, \mu_{\eta}^{W ; K}$ is the image of $\mu_{0}^{W ; K}$ under a continuous map (independent of $K$ ), it follows that for the above sequence $\mu_{\eta}^{W ; K}$ converge also for every $\eta$. We denote the resulting limits by $\mu_{\eta}^{W}$. It is easy to verify that these measures on $\mathscr{P}$ inherit the corresponding versions of the conditions (A.1.7)-(A.1.9) with $K$ stricken out (or taken to $\infty$ ). In particular, (A.1.8) yields

$$
\mu_{T_{x} \eta}^{T_{x} W}(d \rho)=\mu_{\eta}^{W}\left(d T_{-x} \rho\right) .
$$

Remark. In relation to the above statements, one should note that even though $\eta=0$, the measure $\mu_{\eta=0}^{W}$ is affected by the randomness. It is manifested there by the fact that $\mu_{\eta=0}^{W}$ is supported in the set of Gibbs measures whose random couplings $\eta$ are set to zero only in $\Lambda$ !

So far, the construction resulted in objects which are defined for all $\eta$ but have only the finite-volume versions of the desired properties. That limitation may be lifted at the price of a restriction to $v$-a.e. $\eta$. One may either invoke here Doob's martingale convergence theorem, or apply Lemma A.1.1 - by which there exists a sequence $W \rightarrow \infty$, for which $\mu_{\eta}^{W}$ converge to a limit, $\mu_{\eta}$. The consistency condition 
resulting from (A.1.7), implies that the limit reproduces $\mu^{W}$ :

$$
\mu_{\eta}^{W}=E\left(\mu_{\eta} \mid \eta_{W}\right), \text { for all } W \text {. }
$$

Furthermore, (applying Doob's convergence theorem) the pointwise convergence

$$
\mu_{\eta}^{W} \rightarrow \mu_{\eta}
$$

holds not only for the initial sequence, but - with the exclusion of a set of $v$-measure zero, it holds for all the (countably many) sequences of concentric hyper-cubes.

4. It remains to verify that the measure $\mu_{\eta}$ constructed above satisfy the conditions listed in Proposition 5.1. The translation covariance of $\mu_{\eta}$ is implied by (A.1.10) and the translation invariance of the above convergence statement. The transformation law (5.7) is a limiting version of (A.1.9), which holds by the continuity of the maps $U_{\Delta \eta}$.

To prove that for $v$-a.e $\eta, \mu_{\eta}$ is supported in the set of the corresponding Gibbs measures, it suffices to show that for every finite region $\Lambda$ and every local continuous function $f$ of the spins

$$
\int v(d \eta) \int \mu_{\eta}(d \rho)\left|\rho\left(\gamma_{A}^{\eta} f\right)-\rho(f)\right|=0,
$$

where $\gamma_{A}^{\eta}$ is the corresponding Gibbs specification (see [25]). Our bounds on the interaction, discussed in Sect. 3b, easily imply that for $v$-a.e $\eta, \gamma_{A}^{\eta} f$ is a continuous function of the spins, and thus $\rho\left(\gamma_{A}^{n} f\right)$ is a continuous function of $\rho$. Therefore, the integral in (A.1.13) is the limit of the corresponding expressions with $K \rightarrow \infty$ and (in that order) $W \rightarrow \infty$.

By the definition of $\mu_{\eta}^{W ; K}$

$$
\int v(d \eta) \int \mu_{\eta}^{W ; \mathrm{K}}(d \rho)\left|\rho\left(\gamma_{\mathrm{A}}^{\eta} f\right)-\rho(f)\right| \int v(d \eta)\left|\rho_{\eta, \mathrm{K}}\left(\gamma_{\Lambda, \mathrm{K}}^{\eta} f\right)-\rho_{\eta, \mathrm{K}}(f)\right|,
$$

$\rho_{\eta^{\prime}, K}$ being is the p.b. Gibbs state in $[-K, K]^{d}$ with the random couplings $\eta^{\prime}$, and $\gamma_{A, K}^{\eta}$ being the corresponding specification. If the interaction is of finite range then the difference on the right side of (A.1.14) vanishes for large enough $W$. In the case of infinite-range interactions the difference of the first two terms is bounded in absolute value by $\Delta(\eta, \Lambda, K)\|f\|_{\infty}$ with $\Delta(\eta, \Lambda, K)$ being the sum of the absolute values of the interaction terms which directly couple spins in $\Lambda$ with spins in the complement of $[-K, K]^{d}$. Using energy estimates similar to those in Sect. $3 b$, it is easy to produce a uniform bound on this term which vanishes as $K \rightarrow \infty$.

The above arguments imply that the constructed measures have the properties i)-v) listed in Proposition 5.1. Since $\mu_{\eta}$ is supported on Gibbs states, Eq. (3.9) gives

$$
\int v(d \eta) \int \mu_{\eta}(d \rho) \rho\left(g_{\alpha, 0}\right) \in\left[\frac{\partial}{\partial h_{\alpha}-0} F(T,\{h\},\{\varepsilon\}), \frac{\partial}{\partial h_{\alpha}+0} F(T,\{h\},\{\varepsilon\})\right] .
$$

In order to produce the function $\mu_{\eta,+}$ (and $\mu_{\eta,-}$ ) for which the above bound is saturated one may take a weak limit of a sequence of function $\mu^{(n)} \in \mathscr{L}(\mathscr{H}, \mathscr{M})$ with $h^{(n)} \rightarrow h+0$ (and, correspondingly $h^{(n)} \rightarrow h-0$ ). By (A.1.6), such $\mu^{(n)}$ are uniformly continuous, in the sense of a metric associated with the convergence criterion (A.1.1), and the sequence satisfies the conditions of Lemma A.1.1-which implies the existence of a convergent subsequence. It is now elementary to verify that any limit, $\mu \in \mathscr{L}(\mathscr{H}, \mathscr{M})$, inherits the properties i)-iv) of $\mu^{(n)}$. With $h^{(n)} \rightarrow h+0(h-0)$, the 
bounds (A.1.15) yield in the limit:

$$
\int v(d \eta) \int \mu_{\eta}(d \rho) \rho\left(g_{\alpha, 0}\right)=\frac{\partial}{\partial h_{\alpha} \pm 0} F(T,\{h\},\{\varepsilon\}),
$$

which is (5.9).

\section{Appendix II: A General Lower Bound for the Moment Generating Function}

In the proof of the main result (Sect. 6), the most relevant fact about the limiting distribution of the random variable $\tilde{G}_{\Lambda} / \sqrt{|\Lambda|}$ is that it exhibits arbitrarily large fluctuations - on the scale of its second moment. A convenient way to make our argument is by establishing a lower bound on the rate of growth of the moment generating function. In this appendix we derive a general criterion which can be used for such a purpose, presented in a notation which is widely used in the probability literature on the central limit theorem. The correspondence is: $n \leftrightarrow \Lambda, k_{n} \leftrightarrow|\Lambda|, S_{n} \leftrightarrow \tilde{G}_{\Lambda} / \sqrt{|\Lambda|}$, and $\mathscr{F}_{n, i} \leftrightarrow$ the $\sigma$-algebras generated by the "first $i$ " $\eta$ variables in $\Lambda$.

Proposition A.2.1. Let $S_{n, i}$ be a zero-mean martingale array, with respect to the filtration $\mathscr{F}_{n, i}\left(1 \leqq i \leqq k_{n}\right)$; with $S_{n, i}-S_{n, i-1} \equiv X_{n, i}$, and $S_{n, k_{n}} \equiv S_{n}$ (so that $\left.S_{n, i}=E\left(S_{n} \mid \mathscr{F}_{n, i}\right)\right)$. Assume the conditional variation $V_{n}$, and its large-deviation component $U_{n, a}$ :

$$
\begin{aligned}
V_{n} & =\sum_{i=1}^{k_{n}} E\left(X_{n, i}^{2} \mid \mathscr{F}_{n, i-1}\right), \\
U_{n, a} & =\sum_{i=1}^{k_{n}} E\left(X_{n, i}^{2} I\left[\left|X_{n, i}\right|>a\right] \mid \mathscr{F}_{n, i-1}\right),
\end{aligned}
$$

satisfy the following lower bound (a) and the upper bound (b):

a) for some constant $\eta>0$,

$$
\operatorname{Prob}\left(V_{n} \leqq \eta^{2}-\delta\right) \underset{n \rightarrow \infty}{\longrightarrow} 0, \text { for all } \delta>0,
$$

b) for each $a>0$

$$
U_{\cdot n, a} \underset{n \rightarrow \infty}{\stackrel{D}{\longrightarrow}} 0
$$

[which is obviously satisfied if the Lindeberg condition $-E\left(U_{n, a}\right) \underset{n \rightarrow \infty}{\longrightarrow} 0$ holds]. Then, for all $t \in \mathbb{R}$,

$$
\liminf _{n \rightarrow \infty} E\left(e^{t S_{n}}\right) \geqq e^{t^{2} \eta^{2} / 2}
$$

The first step towards the proof is the following elementary statement.

Lemma A.2.2. There is a function $\varepsilon(a)$ - which vanishes as $a \rightarrow 0$, with which

$$
E\left(e^{X}\right) \geqq e^{[1 / 2-\varepsilon(a)] E\left(X^{2} I[|X| \leqq a]\right)}
$$

for any random variable with $E(|X|)<\infty, E(X)=0$. 
Proof. First let us note that some $\tilde{\varepsilon}$, with $\tilde{\varepsilon}(a) \underset{a \rightarrow 0}{\longrightarrow} 0$,

$$
e^{X} \geqq 1+X+\left[\frac{1}{2}-\tilde{\varepsilon}(a)\right] X^{2} I[|X| \leqq a] .
$$

Averaging (A.2.7), and exponentiating the result [with the aid of the fact that $\ln (1+z) / z \underset{z \rightarrow 0}{\longrightarrow} 1]$, one gets (A.2.6).

Proof of Proposition A.2.1. By a scaling argument, it clearly suffices to prove (A.2.5) for $t=1$.

Lemma A.2.2 implies that the random variables

$$
R_{n, k}=\exp \left[\sum_{i=1}^{k} X_{n, i}-\left[\frac{1}{2}-\varepsilon(a)\right] \sum_{i=1}^{k} E\left(X_{n, i}^{2} I\left[\left|X_{n, i}\right| \leqq a\right] \mid \mathscr{F}_{n, i-1}\right)\right]
$$

form a submartingale, i.e.

$$
E\left(R_{n, k} \mid \mathscr{F}_{n, k-1}\right) \geqq R_{n, k-1}, \quad \text { for } \quad k=1, \ldots, k_{n} .
$$

Consequently,

$$
1 \leqq E\left(R_{n, 1}\right) \leqq \cdots \leqq E\left(R_{n, k_{n}}\right)=E\left(e^{S_{n}-[1 / 2-\varepsilon(a)]\left(V_{n}-U_{n, a}\right)}\right) .
$$

Applying the Hölder inequality with $q \geqq 1,(1 / q)+\left(1 / q^{*}\right)=1$, we get

$$
1 \leqq E\left(e^{q S_{n}}\right)^{1 / q} E\left(e^{-[1 / 2-\varepsilon(a)]\left(V_{n}-U_{n, a}\right) q^{*}}\right)^{1 / q^{*}} .
$$

Conditions (A.2.3) and (A.2.4), and the positivity of $V_{n}-U_{n, a}$, imply - for the last factor in (A.2.11), that

$$
\lim _{a \rightarrow 0} \limsup _{n \rightarrow \infty} E\left(e^{-[1 / 2-a(\varepsilon)]\left(V_{n}-U_{n, a}\right) q^{*}}\right)^{1 / q^{*}} \leqq e^{-1 / 2 \eta^{2}}
$$

for each $q^{*}<\infty$. Hence, for each $q>1$,

$$
\liminf _{n \rightarrow \infty} E\left(e^{q S_{n}}\right) \geqq e^{q \eta^{2} / 2},
$$

and by scaling-

$$
\liminf _{n \rightarrow \infty} E\left(e^{t S_{n}}\right) \geqq t^{t^{2} \eta^{2} /(2 q)}
$$

Taking $q \downarrow 1$ we get (A.2.5).

Remark. It may be added that if the assumption (A.2.3) of Proposition A.2.1 holds with $\eta^{2}=\lim \sup E\left(S_{n}^{2}\right)$ (which is the largest value at which it can hold) then $S_{n}$ converges, in distribution, to a Gaussian. That case is already covered by the following version of the Central Limit Theorem (which incorporates improvements due to a large number of contributors).

Proposition A.1.3. ([30, Theorem 3.2]). If a zero-mean martingale (double) array $S_{n, i}$, with the rest of the notation of Proposition A.2.1, satisfies:

$\left.a^{\prime}\right)$ for some constant $b, V_{n} \stackrel{P}{\rightarrow} b^{2}$ i.e.

$$
\operatorname{Prob}\left(\left|V_{n}-b^{2}\right| \geqq \delta\right) \underset{n \rightarrow \infty}{\longrightarrow} 0, \text { for all } \delta>0,
$$


and b) the conditional Lindeberg property (A.2.4), then

$$
S_{n} \stackrel{d}{\rightarrow} N(0, b) .
$$

We apply this Theorem in the proof of (1.2), in Sect. 6. Though, as explained there, for the main result of this paper it suffices to have Proposition A.2.1.

\section{Appendix III: Variance Lower Bounds for Probability Measures on $\mathbb{R}^{\mathbf{N}}$}

a. Measures on $\mathbb{R}$. In this appendix we prove the main properties of the functions $\theta_{v}(\cdot)$ and $\gamma_{v}(\cdot)$ which were used in Sect. 6 and 7. [In the process, we correct a statement made in ref. [3] - where $\theta_{v}$ was mentioned only with $T=0$ and the absolute continuity is mistakenly replaced by a mere continuity requirement.] These functions provide uniform lower bounds on the variance of quantities dependent on the random parameters, on the basis of information concerning their derivatives.

The relevant constraints on the derivatives may be introduced in terms of the Lipschitz seminorm $\|g\| \equiv \sup _{x, y} \frac{|g(x)-g(y)|}{|x-y|}$. We denote by $\mathscr{V}_{1, \beta}$ the following sets of functions (on $\mathbb{R}$ ):

For $\beta<\infty$ :

$$
\mathscr{V}_{1, \beta}=\left\{g \in C^{1}(\mathbb{R})|| g^{\prime}(\cdot)\left|\leqq 1,\left\|g^{\prime}\right\|\right| \leqq \beta\right\} .
$$

For $\beta=\infty$ :

$$
\mathscr{V}_{1, \infty}=\{g \in C(\mathbb{R})|\|||| \mid \leqq 1\} .
$$

The elements of $\mathscr{V}_{1, \infty}$ are absolutely continuous, and hence $\ell$ [Lebesgue measure]a.e. differentiable, with $g(h)-g(0)=\int_{0}^{h} g^{\prime}(z) d z$. The derivative $g^{\prime} \in L^{\infty}(d \ell)$ satisfies $\left\|g^{\prime}\right\|_{\infty} \leqq 1$, which is akin to the bound $\left|g^{\prime}(\cdot)\right| \leqq 1$ seen in the definition of $\mathscr{V}_{1, \beta}$.

The functions $\theta_{v}(\cdot)$ and $\gamma_{v}(\cdot)$ (the latter being relevant when $g(\cdot)$ is monotone in $\eta$ ) are defined as follows, for $M \in[-1,1]$ and $T \geqq 0$,

$$
\begin{aligned}
& \theta_{v}(M, T)=\inf \left\{\left[\int g(\eta)^{2} v(d \eta)\right]^{1 / 2} \mid g \in \mathscr{V}_{1,1 / T}, \int g^{\prime}(\eta) v(d \eta)=M\right\}, \\
& \gamma_{v}(M, T)=\inf \left\{\left[\int g(\eta)^{2} v(d \eta)\right]^{1 / 2} \mid g \in \mathscr{V}_{1,1 / T}, g^{\prime}(\cdot) \geqq 0, \int g^{\prime}(\eta) v(d \eta)=M\right\} .
\end{aligned}
$$

It should be noted that the $L^{2}(d v)$ norm of $g$ may be replaced in the above definitions by the square-root of the variance of $g$ with respect to $d v$. Of particular interest for us are sufficient conditions for the strict positivity of these uniform bounds. Before turning to this issue, let us make a couple of preliminary observations.

Propositions A.3.1. For each Borel probability measure v(on $\mathbb{R})$ :

i. $\theta_{v}(M, T)$ and $\gamma_{v}(M, T)$ are convex in $M$, for any $T \geqq 0$, with $\theta_{v}(\cdot, T)$ an even function (while $\gamma_{v}(\cdot, T)$ is defined only for $M \geqq 0$ ).

ii. $\theta_{v}(M, T) / M$ and $\gamma_{v}(M, T) / M$ are nondecreasing in $|M|$, and

$$
\theta_{v}(M, T) \leqq \gamma_{v}(M, T) \leqq|M| \operatorname{Av}\left(\eta^{2}\right)^{1 / 2} .
$$


If $v$ is absolutely continuous, with respect to $\ell$, with $v(d \eta)=\varphi(\eta) d \eta$, then

$$
\theta_{v}(M, T) \geqq \frac{|M|}{2\left[\int\left|\frac{d}{d h} \sqrt{\varphi(h)}\right|^{2} d h\right]^{1 / 2}} .
$$

The convexity is by the affinity of the constraint, and the convexity of the norm of $L^{2}(d v)$. More explicitly: for $M=(1-\lambda) M_{0}+\lambda M_{1}$ one can take as variational functions $g=(1-\lambda) g_{0}+\lambda g_{1}$, with $g_{i}$ satisfying the corresponding constraints. That yields: $\theta_{v}(M) \leqq(1-\lambda) \theta_{v}\left(M_{0}\right)+\lambda \theta_{v}\left(M_{1}\right)$, and the similar inequality for $\gamma_{v}(M)$. Statements made in ii. are derived by the natural arguments, omitted here because of their simplicity and the fact that (A.3.3)-(A.3.4) are not used in this work. Let us note, however, that (A.3.4) is derived by ignoring all the constraints on the derivatives.

Most important for the arguments made in Sect. 6 is to know under what conditions on $v$, does $\theta_{v}(M, T)=0$ imply $M=0$. The following proposition answers this question.

\section{Proposition A.3.2. For Borel probability measures (v) on $\mathbb{R}$ :}

i. At $T=0 ; \theta_{v}(M, 0)$ vanishes only at $M=0 \Leftrightarrow v$ is absolutely continuous with respect to the Lebesgue measure $(\ell)$.

ii. For $T>0 ; \theta_{v}(M, T)$ vanishes only at $M=0 \Leftrightarrow v$ has no isolated point masses (i.e. points with $v(\{h\}) \neq 0$ and $v([h-a, h) \cup(h, h+a])=0$ for some $a>0)$.

iii. For $T>0 ; \gamma_{v}(M, T)$ vanishes only at $M=0 \Leftrightarrow v$ is not concentrated at a single point.

Proof. i. $\Leftarrow$ (at $T=0$ ): In the absolutely continuous case, the infimum in (A.3.2) is actually attained in the set $\mathscr{V}_{1, \infty}$. To prove that statement, we note that $v$ is of the form $v(d h)=\varphi(h) d h$, with $\varphi(h) \in L^{1}(d h)$. Let $g_{n}$ be a minimizing sequence for a given $M \neq 0 .\left|g_{n}(0)\right|$ is uniformly bounded for such a sequence, as can be easily deduced from the bound $\left|g_{n}(h)\right| \geqq\left.|| g_{n}(0)|-| h\right|_{+}$. By the sequential $w^{*}$-compactness of the unit ball of $L^{\infty}(d h)$, there is a subsequence, for which both $g_{n}(0)$ and the derivatives converge; $g_{n}^{\prime} \rightarrow f \in L^{\infty}(\mathrm{dh})$ (in the $w^{*}$ sense). It follows that $g_{n}$ converge pointwise to a function $g \in \mathscr{V}_{1, \infty}$, with $g^{\prime}=f(\ell$-a.e.). For the limiting functions we have (using the Fatou lemma, and the fact that $g_{n}$ was a minimizing sequence)

a. $\int f(h) \varphi(h) d h=M, \quad$ b. $\int g^{2}(h) \varphi(h) d h=\theta_{v}(M, 0)$.

In the above notation, if $x$ is in the Lebesgue sets [31] of $\varphi$ and $g^{\prime}$, with $\varphi(x) \cdot g^{\prime}(x) \neq 0$ (and, since $M \neq 0$, the set of such points has positive $\ell$-measure) then for some $a>0, g$ does not vanish is the neighborhood $(x-a, x+a)$ except possibly at one point, while $\varphi$ is nonzero on a positive measure subset of $(x-a, x+a)$. Hence $\theta_{v}(M, 0)>0$.

$\Rightarrow($ at $T=0)$ : If $v$ is not absolutely continuous with respect to $\ell$, there is a bounded measurable set $S$ with $\ell(S)=0$ and $v(S)>0$. Since $C(\mathbb{R})$ is dense in $L^{1}$ of the Borel measure $(d v+d \ell)$, there is a sequence of continuous functions $f_{n}$, with $\left\|f_{n}-I[S]\right\|_{L^{1}(d v+d l)} \rightarrow 0\left(I[S]\right.$ being the indicator function). Let $g_{n}(h)=\int_{0}^{h} f_{n}(z) d z$. 
Then

and

$$
\int g_{n}^{\prime}(h) v(d h)=\int f_{n}(h) v(d h) \rightarrow v(S)
$$

$$
\int g_{n}^{2}(h) v(d h) \rightarrow 0
$$

since $g_{n}(h) \rightarrow \ell([0, h] \cap S)=0$, uniformly in $h$. It follows that $\theta_{v}(M, 0)=0$ for all $|M|<v(S)$.

ii. $\Leftarrow$ At $T>0$, compactness arguments similar to those used in ii., but applied to $g^{\prime \prime}$, show that for each $M, \theta_{v}(M, T)$ is realizable by means of a certain function $g \in C^{1}$, with

a. $\int g^{\prime}(h) v(d h)=M, \quad$ b. $\int g^{2}(h) v(d h)=\theta_{v}(M, 0)$.

Let $S$ be the support of $v$ (the smallest closed set with $v\left(S^{c}\right)=0$ ). If $\theta_{v}(M, T)=0$ then $g \equiv 0$ on $S$. The assumption on $v$ is equivalent to the statement that $S$ has no isolated points. The vanishing of $g$ on such a set implies $g^{\prime} \equiv 0$ on $S$, and that can occur only if $M=0$.

$\Rightarrow$ For $v$ with an isolated point $(h)$, it is easy to construct $g$ which show that $\theta_{v}(M, T)$ vanishes at a certain positive value of $M$, depending only on $v(\{h\})$ and the distance of $h$ to $S \backslash\{h\}$.

iii. For $T>0, \gamma_{v}(M, T)$ is attainable by an analog of (A.3.7) - with $g$ restricted to be monotone, as well as to have a continuous derivative. If $\gamma_{v}(M, T)=0$ then $g$ vanishes identically on $S=\operatorname{supp} v$. If also $M \neq 0$, then $g^{\prime}(x) \neq 0$ for at least one point $x \in S$, with $g(x)=0$. That, however, implies $g^{2}>0$ everywhere else, which is in contradiction with the vanishing of $\gamma$.

b. Measures on $\mathbb{R}^{N}$. In Sect. 7 we need a finite dimensional extension of the above result - to functions of a vector valued random variable $h$, with values in $\mathbb{R}^{N}$. It turns out that in order to obtain a lower bound on the variance of $g$, on the basis of $M=\operatorname{Av}\left(\partial g / \partial \eta_{1}\right)$, it suffices to have upper bounds on the derivatives of $g$ with respect to just $\eta_{1}$.

To express these bounds let us denote the points in $\mathbb{R}^{N}$ as $\underline{h}=\left(h_{1}, h_{\perp}\right)$, and let $\|\mid g\|_{1} \equiv \sup \left(\left|g(h)-g\left(h^{\prime}\right)\right|\right) /\left(\left|h_{1}-h_{1}^{\prime}\right|\right)$. We define the space $\mathscr{V}_{1, \beta}^{(\bar{N})}$ and the function $h_{\perp}=h_{\perp}^{\prime}$

$\theta_{v}^{(N)}(M, T)$ by analogy with (A.3.1) and (A.3.2), with $g^{\prime}$ replaced by $\left(\partial g / \partial h_{1}\right)$ and $\|\mid g\|$ by $\|\mid g\|_{1}$.

Lemma A.3.3. Let $v(d h)$ be a probability measure on $\mathbb{R}^{N}$, written as:

$$
v(d h)=\int v_{\perp}\left(d h_{\perp}\right) v\left(d h_{1} \mid h_{\perp}\right)
$$

(i.e. $v_{\perp}\left(d h_{\perp}\right)$ is the projection of $v$ on $\mathbb{R}^{N-1}$, and $v\left(\cdot \mid h_{\perp}\right)$ is the conditional probability). If, at some $T \geqq 0$, the one dimensional measures $v_{h_{\perp}}(\cdot) \equiv v\left(\cdot \mid h_{\perp}\right)$ satisfy

$$
\theta_{h_{\perp}}(M, T) \equiv \theta_{v_{h_{\perp}}}(M, T) \neq 0 \text { for every } M \neq 0
$$

for $v$-almost every $h_{\perp}$, then $\theta_{v}^{(N)}(M, T) \neq 0$ for all $M \neq 0$.

Proof. For $g \in \mathscr{V}_{1, \beta}^{(N)}$, satisfying

$$
\int_{\mathbf{R}^{N}} \frac{\partial g}{\partial h_{1}} v(d h)=M
$$


let us denote

$$
m\left(h_{\perp}\right)=\int_{\mathbf{R}} \frac{\partial g}{\partial h_{1}} v_{h_{\perp}}\left(d h_{1}\right) .
$$

Since $M \leqq(M / 2)+1 \cdot v_{\perp}\left(\left\{m\left(h_{\perp}\right) \geqq M / 2\right\}\right)$, we have

$$
v_{\perp}\left(\left\{h_{\perp} \in \mathbb{R}^{N-1} \mid m\left(h_{\perp}\right) \geqq M / 2\right\}\right) \geqq M / 2 \text {. }
$$

Hence

$$
\begin{aligned}
\int_{\mathbf{R}^{N}} g^{2} v(d h) & =\int\left[\int g^{2}\left(h_{1}, h_{\perp}\right) v_{h_{\perp}}\left(d h_{1}\right)\right] v_{\perp}\left(d h_{\perp}\right) \\
& \geqq \int \theta_{h_{\perp}}^{2}\left(m\left(h_{\perp}\right), T\right) v_{\perp}\left(d h_{\perp}\right) \\
& \geqq \inf \left\{\int_{A} \theta_{h_{\perp}}^{2}(M / 2, T) v_{\perp}\left(d h_{\perp}\right) \mid A \subset \mathbb{R}^{N-1}, v_{\perp}(A) \geqq M / 2\right\} \\
& =\int \theta_{h_{\perp}}^{2}(M / 2, T) I\left[\theta_{h_{\perp}}(M / 2, T) \leqq s\right] v_{\perp}\left(d h_{\perp}\right),
\end{aligned}
$$

where $s$ is determined by the condition

$$
\int I\left[\theta_{h_{\perp}}(M / 2, T) \leqq s\right] v_{\perp}\left(d h_{\perp}\right)=M / 2 .
$$

Since the above bound holds for each $g \in \mathscr{V}_{1, \beta}^{(N)}$ satisfying (A.3.10), we get

$$
\theta_{v}^{(N)}(M, T) \geqq \int \theta_{h_{\perp}}^{2}(M / 2, T) I\left[\theta_{h_{\perp}}(M / 2, T) \leqq s\right] v_{\perp}\left(d h_{\perp}\right),
$$

which is strictly positive for each $M \neq 0$ (by the nonvanishing of $\theta_{\mathrm{h}_{\perp}}^{2}(M / 2, T)$ ).

Combining this criterion with Proposition A.3.2, we get the following corollary - which is used in our result on the continuous symmetry breaking.

Corollary A.3.4. For rotation-invariant Borel probability measures (v) on $R^{N}$ :

i) If $v$ does not have isolated spheres of positive measures (i.e. if the radial distribution of $v$ does not have isolated point masses), then $\theta_{v}^{(N)}(M, T) \neq 0$ for every $M \neq 0$ and $T>0$.

ii) If, in addition, $v$ is absolutely continuous with respect to the Lebesgue measure on $R^{N}$, then $\theta_{v}^{(N)}(M, T=0)=0$ only at $M=0$.

Acknowledgements. We thank J. Bricmont, D. Fisher, T. Hara, P. Hitczenko, T. Spencer, D. Stroock, and $\mathrm{H}$. Tasaki for stimulating discussions; their comments prodded us to find improvements in the analysis and clarify the relation of this work with a number of topics concerning random systems. M. A. also thanks T. Spencer for hospitality at the Institute for Advanced Study, and J. W. is grateful to Rutgers University's Graduate School (NB) for the Academic Excellence Fellowship awarded to him.

\section{References}

1. Imry, Y. and Ma, S. -K.: Random-field instability for the ordered state of continuous symmetry. Phys. Rev. Lett. 35, 1399 (1975)

2. Balian, R., Maynard, R., Toulouse, G. (eds.): Ill-condensed matter. Amsterdam: North-Holland (1979); Osterwalder, K. Stora, R. (eds.): Critical phenomena, random systems, gauge theories, vol. II. Amsterdam: North-Holland (1986); Fisher, D. S., Grinstein, G. M., Khurana, A.: Theory of random magnets, Physics Today, vol. 14, \#12, p. 56, Dec. 1988 
3. Aizenman, M. Wehr, J.: Rounding of first-order phase transitions in systems with quenched disorder. Phys. Rev. Lett. 62, 2503 (1989)

4. Chalker, J.: On the lower critical dimensionality of the Ising model in a random field. J. Phys. C 16, 6615 (1983); Fisher, D., Fröhlich, J., Spencer, T.: The Ising model in a random magnetic field. J. Stat. Phys. 34, 863 (1984)

5. Imbrie, J. Z.: Lower critical dimension of the random-field Ising model. Phys. Rev. Lett. 53, 1747 (1984), and: The ground state of the three-dimensional random-field Ising model. Commun. Math. Phys. 98, 145 (1985)

6. Bricmont, J. Kupiainen, A.: Lower critical dimension for the random-field Ising model. Phys. Rev. Lett. 59, 1829 (1987), and: Phase transition in the 3D random field Ising model. Commun. Math. Phys. 116, 539 (1988)

7. Wu, F. Y.: The Potts model. Rev. Mod. Phys. 54, 235 (1982); Kotecky, R., Shlosman, S. B.: First-order phase transitions in large entropy lattice models. Commun. Math. Phys. 83, 493 (1982)

8. Stinchcombe, R. B.: In: Phase transitions and critical phenomena, vol. 7. Domb, C., Lebowitz, J. (eds.) London: Academic Press 1983; Chayes, J. T., Chayes, L., Fröhlich, J.: The low-temperature behavior of disordered magnets. Commun. Math. Phys. 100, 399 (1985; Aizenman, M., Chayes, J. T., Chayes, L., Newman, C. M.: The phase boundary in dilute and random Ising and Potts ferromagnets. J. Phys. A (Math. Gen.) 20, L313 (1987)

9. Derrida, B. Shnidman: Y.: Possible line of critical points for a random field Ising model in dimension 2. J. Phys. Lett. 45, L577 (1984); Grinstein, G., Fernandez, J.: Equilibration in random-field Ising systems. Phys. Rev. B 29. 6389 (1984)

10. Hui, K., Berker, A. N.: Random field mechanism in random bond multicritical systems. Phys. Rev. Lett. 62, 2507 (1989)

11. Cox, U. J., Gibaud, A., Cowley, R. A.: Effect of impurities on the first-order phase transition of $\mathrm{KMnF}_{3}$. Phys. Rev. Lett. 61, 982 (1988)

12. Imry, Y.: Random External Fields. J. Stat. Phys. 34, 849 (1984); Grinstein, G.: On the lower critical dimension of the random-field Ising model. J. Appl. Phys. 55, 2371 (1984); Nattermann, T., Villain, J.: Random-field Ising systems: a survey of current theoretical views. Phase Transitions 11, 1 (1988)

13. Binder, K., Young, A. P.: Spin glasses: Experimental facts, theoretical concepts, and open questions. Rev. Mod. Phys. 54, 801 (1986); Fisher, D., Huse, D.: Absence of many states in realistic spin glasses. J. Phys. A: Math. Gen. 20, L1005 (1987) and: Equilibrium behavior of the spin-glass ordered phase. Phys. Rev. B 38, 386 (1988); Bovier, A., Fröhlich, J.: A heuristic theory of the spin glass phase. J. Stat. Phys. 44, 347 (1986)

14. Wehr, J., Aizenman, M.: Fluctuations of extensive functions of quenched random couplings. To appear in J. Stat. Phys.

15. Ruelle, D.: Probability estimates for continuous spin systems. Commun. Math. Phys. 50, 189 (1976); Lebowitz, J., Presutti, E.: Statistical mechanics of unbounded spins. Commun. Math. Phys. 50, 195 (1976).

16. Griffiths, R., Lebowitz, J.: Random spin systems: some rigorous results. J. Math. Phys. 9, 1284 (1968); Ledrappier, F.: Pressure and variational principle for random Ising model. Commun. Math. Phys. 56, 297 (1977); Vuillermot, P.: Thermodynamics of quenched random spin systems and application to the problem of phase transitions in magnetic (spin) glasses. J. Phys. A. (Math. Gen.) 10, 1319 (1977); Pastur, L. A.: Figotin, A. L.: Theory of disordered spin systems. Theor. Math. Phys. 35, \#2, 193 (Russian) [403-transl.] (1978)

17. Ruelle, D. Statistical mechanics. Rigorous results. New York: Benjamin 1969; Israel, R.: Convexity in the theory of lattice gases. Princeton, NJ: Princeton University Press 1978

18. Fortuin, C., Kasteleyn, P. Ginibre, J.: Correlation inequalities on some partially ordered sets. Commun. Math. Phys. 22, 89 (1971)

19. Oliviera, S. M., Oliviera, P. M., Continentino, M. A.: Site diluted antiferromagnet in a uniform field. Physica A 152, 477 (1988); de Quiroz, S. L. A., dos Santos, R. R.: The dilute Ising antiferromagnet in a uniform field on a square lattice. J. Phys. C 21, 1995 (1988)

20. Mermin, D., Wagner, H.: Absence of ferromagnetism or anti-ferromagnetism in one- or twodimensional isotropic Heisenberg models. Phys. Rev. 17, 1133 (1966)

21. Herring, C. H., Kittel, C.: On the theory of spin waves in ferromagnetic media. Phys. Rev. 81, 869 (1951) 
22. Pfister, C.: On the symmetry of the Gibbs states in two-dimensional lattice systems. Commun. Math. Phys. 79, 181 (1981)

23. Dobrushin, R. L., Shlosman, S. B.: Absence of breakdown of continuous symmetry in twodimensional models of statistical mechanics. Commun. Math. Phys. 42, 31 (1975)

24. Fannes, M., Vanheuverzwijn, P., Verbeure, A.: Energy-entropy inequalities for classical lattice systems. J. Stat. Phys. 29, 547 (1982)

25. Georgii, H.: Gibbs measures and phase transitions. Berlin, New York: De Gruyter 1988

26. Chevalley, C.: Theory of Lie groups, Vol. I. Princeton, NJ: Princeton University Press 1946

27. Billingsley, P.: Convergence of probability measures. New York: John Wiley 1968; Kelley, J.: General Topology. New York: Van Nostrand 1955

28. Reed, M., Simon, B.: Methods of modern mathematical physics, vol. 1. New York: Academic Press 1981

29. Doob, J. L.: Stochastic processes. New York: John Wiley 1953

30. Hall, P., Heyde, C. C.: Martingale limit theory and its application. New York: Academic Press 1980

31. Stein, E. M., Weiss, G.: Fourier analysis on Euclidean spaces. Princeton, NJ: Princeton University Press 1971

Communicated by A. Jaffe

Received October 26, 1989 